\title{
$\begin{array}{ll}\text { Research Square } & \text { Preprints are preliminary reports that have not undergone peer review. } \\ \text { They should not be considered conclusive, used to inform clinical practice, }\end{array}$ or referenced by the media as validated information. \\ Leaf Transcriptome Analysis of Medicago \\ Ruthenica, Revealing Its Response and Adaptive Strategy to Drought Stress and Rehydration
}

\section{Rina Wu}

Inner Mongolia Agricultural University https://orcid.org/0000-0002-3787-3230

\section{Bo Xu}

Inner Mongolia Agricultural University

Fengling Shi ( $\nabla 1072948345 @ q q . c o m$ )

College of Grassland Resources and Environment, Key Laboratory of Grassland Resources of the Ministry of Education, Inner Mongolia Agricultural University, Hohhot 010011, China

\section{Research article}

Keywords: Medicago ruthenica(L.)cv.Zhilixing, Dourght stress, Rehydration, Transcriptome, Adaptive strategy, Differentially expressed genes

Posted Date: September 8th, 2020

DOI: https://doi.org/10.21203/rs.3.rs-63066/v1

License: (9) (1) This work is licensed under a Creative Commons Attribution 4.0 International License. Read Full License 


\title{
Leaf transcriptome analysis of Medicago ruthenica, revealing its response and adaptive strategy to drought stress and rehydration
}

\author{
Rina $\mathrm{Wu}^{1}$, Bo Xu${ }^{1}$, Fengling Shi ${ }^{1 *}$
}

\begin{abstract}
Background: Recently, drought stress has brought tremendous loss on the production of agriculture and animal husbandry. In realistic production, plants are often in cyclic wet-dry environment. Therefore, the factors that affect the final yield of plants in adversity including the resistance and tolerance to drought and the ability of plants to resume from the previous damage after rehydration. So it's necessary for us to study the response and adaptive strategies of plants to drought and rehydration. Generally, the yield of herbage with strong resistance is relatively low. However, Medicago ruthenica(L.)cv.Zhilixing has the advantages of strong resistance and high yield concurrently. This made it can be used for raising livestock, natural grassland improvement, as a good parent for breeding and a new and high quality resource of stress resistance genes. Now, there are still many problems need to be solved when compared with other important legume forages. Therefore, we analyzed the changes of Medicago ruthenica(L.)cv.Zhilixing on transcription level under drought stress and rehydration, explored its phased response strategies.
\end{abstract}

Results: We obtained 191 DEGs in drought stress, and the three treatments has 43 DEGs in common. Galactose metabolism, Starch and sucrose metabolism, Arginine and proline metabolism,

\footnotetext{
* Correspondence: 1072948345@qq.com

${ }^{1}$ College of Grassland Resources and Environment, Key Laboratory of Grassland Resources of the Ministry of Education, Inner Mongolia Agricultural University, Hohhot 010011, China
} 
TCA cycle, Photosynthesis-antenna proteins, were involved in the adaptation of Medicago ruthenica to 9 days of drought stress. The regulation of Arginine and proline metabolism, Cysteine and methionine metabolism, Photosynthesis-antenna proteins, Ascorbate and aldarate metabolism were conducive to the resistance of Medicago ruthenica to severe drought stress. The regulation of Starch and sucrose metabolism, Flavonoid biosynthesis, Valine, leucine and isoleucine degradation, Circadian rhythm-plant was beneficial to the post drought recovery of Medicago ruthenica.

Conclusions: We preliminarily analyzed the adaptation mechanism of the plant under different drought and rehydration conditions. Medicago ruthenica(L.)cv.Zhilixing adopts different strategies to adapt to different degrees of drought stress and rehydration. The research discovered the genes that can be used as candidate genes to improve stress resistance and drought adaptability of plants. Our transcriptome data dramatically enriches the resources of stress resistance genes. It can provide theoretical support for further adaptation mechanism research of the plant under different drought and rehydration conditions.

Key words: Medicago ruthenica(L.)cv.Zhilixing, Dourght stress, Rehydration, Transcriptome, Adaptive strategy, Differentially expressed genes

\section{Background}

Plants are threatened by various biological or abiotic factors in the process of growth and development. So it's necessary for us to study the stress resistance of plants. Recently, due to environmental degradation, climate warming and water resources shortage, the plant growth 
limited by drought has became a general phenomenon, and the drought stress made huge adverse effect on agricultural-stock production. Therefore, it is extremely valuable to study the response and adaptation strategies of plants to arid environment. The adaptation of plants to drought is reflected in two aspects: the survival ability under drought stress and the rapidly recovery after rehydration[1]. The degree of drought stress increases gradually with the extension of time, and the response of plants to drought also changes constantly, which produces great difficulties to the research in this field. In order to solve this problem, we need to know variation characteristics of plant response to drought with the increasing stress. Moreover, in actual production, plants are often in cyclic wet-dry environments. The resistance and tolerance ability of plant under drought stress is not the sole factor that affects the final yield of plants in the arid environment. The ability of plants to resume normal growth from previous damage after the improvement of water condition is also count for much. Therefore, Chen[2] put forward the concept of drought adaptability, that is, the whole performance of plants in the process of drought and rehydration, which includes both drought resistance ability and recovery ability after rehydration. Whether they can make up for the damage suffered before during rehydration soon is an important performance of plant adaptability to drought stress. They found that the drought adaptability was more affected by the recovery ability through the research on drought adaptability of several maize varieties.

Medicago ruthenica, belongs to the genus Medicago, Leguminosae, is widely distributed in alpine grassland, typical grassland, desertification grassland and has the advantages of cold, drought, salt and alkali resistance, barren resistance, trample resistance, and so on[3,4]. Li[5] compared Medicago ruthenica with other legume forages with strong resistance, and found that the thickness of palisade tissue and sponge tissue and the tightness of palisade tissue structure of 
Medicago ruthenica was the highest. Its drought resistance was not only better than alfalfa, but also stronger than that of Lotus japonicus, Medicago falcata, Lespedeza bicolor, and Trifolium lupinaster. It has great potential in many fields such as grassland improvement, ecological management and grass industry development, and has extremely important ecological significance[6,7].

Simultaneously, it not only has higher nutritional value and good palatability, but also has higher nutritional utilization efficiency than alfalfa[8]. It has no saponin[9]. Surfeit ingestion of this forage will not cause tympanites in domestic animals. Thus, it can be used as a high quality protein feed and has important production significance. However, its stem is inclined and the grass yield and seed yield are lower than alfalfa, which limits the development and utilization of Medicago ruthenica. Therefore, cultivating new Medicago ruthenica varieties with high grass yield and seed yield to meet the production demand is of great practical significance for ecological construction and animal husbandry development. We selected Medicago ruthenica (L.) cv. Zhilixing as the experimental material. It was cultivated by scholars from Inner Mongolia Agricultural University with multiple mixed selection method. And in December 1992, the Chinese Herbage Varietal Resources Registration Board approved and registered it as a bred variety. In addition to its high forage value and strong resistance, Medicago ruthenica (L.) cv. Zhilixing also has higher grass and seed yield than wild-type Medicago ruthenica[10]. The plant is upright and convenient for mowing and cutting. Its green period was from 180 to 200 days. This variety has the characters of high water utilization efficiency and strong tolerance to adversity which made it suitable to cultivate in arid and cold areas.

Shi[11] observed and analyzed the morphological and anatomical structure of this breed. It was 
found that the leaves were elliptic with serrated edges, and the upper epidermis was sparsely pubescent, while the lower epidermis was more abundant than the upper epidermis. The stomatal depression was deeper than that of alfalfa, and the stomatal density, the compactness of palisade tissue and spongy tissue, and the layer number of palisade tissue were also higher than that of alfalfa, which was conducive to photosynthesis and prevented water evapotranspiration in drought. Meanwhile, compared with alfalfa, the leaf veins were more obvious and dense, the conducting tissue was developed, and the intercellular space was smaller. Morphological and anatomical analysis showed that the erect type of Medicago ruthenica was more drought resistant than alfalfa. Furthermore, this variety is not only a good parent for breeding, but also a good gene resource for improving the stress resistance of alfalfa and other forages. It is better than alfalfa in adaptability and resistance. Therefore, it is of great significance on the research, development and utilization of the plant.

Using transcriptome sequencing technology to study the expression of the whole genome in plants under drought stress is helpful to reveal the molecular regulation mechanism of plant response to drought. It is of vital importance to identify the key genes in various metabolic pathways, to explore new genes that related to drought resistance and to construct transcriptional regulatory networks in plant response to stress[12]. RNA-seq has been used to study the response of Medicago ruthenica to single stress for 3 hours (ABA, low temperature, salt stress, etc.)[13]. However, there is no report on the transcriptional changes of the forage under different levels of drought stress and rehydration. Therefore, the experiment aims to study the variations of the molecular level of Medicago ruthenica (L.) cv. Zhilixing under different intensity of drought stress and rehydration. It's benefit to understand the molecular response of the plant to drought stress 
and rehydration, and also to provide a reference for the later study of the phased response strategy of Medicago ruthenica to drought stress and rehydration. The research will provide a new idea for improving the drought resistance and drought adaptability of alfalfa and other forages. And also can provide theoretical support for the development of grass industry and regional vegetation construction in arid and semi-arid areas.

\section{Results}

\section{De-novo assembly transcriptome}

12 cDNA libraries( 6 drought treatments, 3 rehydrations, $3 \mathrm{CKs}$ ) with error rates $<0.1 \%$ were obtained from the transcriptome sequencing of Medicago ruthenica leaves. There were $628,999,764$ clean reads after removing low-quality sequences(Table 1). A total of 712,926 transcripts were obtained by de-novo splicing of data with Trinity software. Taking the longest transcripts as unigene, 308,449 unigenes with an N50 of 1,258 bp were further obtained. The maximum, minimum and average lengths were 13,571 bp, 201 bp and 922 bp, respectively (Fig. 1). The percentage of unigenes with length of $200-500 \mathrm{bp}, 500-1 \mathrm{k} \mathrm{bp}, 1 \mathrm{k}-2 \mathrm{k}$ bp and longer than $2 \mathrm{k}$ bp in total unigenes were $34.63 \%(106,812), 32.91 \%(101,494), 23.98 \% \quad(73,961)$ and $8.49 \%(26,182)$, respectively.

\section{Sequence annotation}

Function annotation for 308,449 unigenes that finally assembled. 198,437 (64.33\%), 244,144 (79.15\%), 76,570 (24.82\%), 145,469 (47.16\%), 133,570 (43.3\%), 141,360 (45.82\%) and 60,332 (19.55\%) unigene sequences were significantly matched to NR, NT, KO, Swiss prot, Pfam, GO 
and KOG database respectively(Fig. 2). There were 30,739 unigenes obtained annotation information in all databases, accounting for $9.96 \%$ of the total unigenes. The number of genes annotated successfully in at least one of the seven databases was 271,580 , accounting for $88.04 \%$ of the total number of unigenes. In unigene's NR database annotation, $64.33 \%$ of the sequences were well matched to the known genes in plants $\left(\mathrm{E}\right.$ value $\left.<10^{-5}\right) .61 .7 \%$ of the sequences were more than $80 \%$ similar to the known sequences, and nearly $80.2 \%$ of the sequences were matched to the following five species sequences: Medicago truncatula(69.4\%), cicer arietinum(6.5\%), Glycine max(1.9\%), Glycine soja(1.4\%), Hordeum vulgare (1.1\%) (Fig. 3).

\section{Identification and analysis of DEGs under drought stress and rehydration}

Compared with CK, 2,905 DEGs were identified by padj $<0.05$. There were 2,221 DEGs in the whole process of drought stress, among which 1,923 were identified in A vs B (CK vs drought stress 9d), including 1,411 up-regulated genes and 512 down-regulated genes; 489 DEGs were identified in A vs C (CK vs drought stress 12d), including 226 up-regulated genes and 263 down-regulated genes; 924 DEGs were identified in A vs D (CK vs rehydration), including 387 up-regulated genes and 537 down-regulated genes. The results showed that the number of DEGs was the most in A vs B when compared with other 2 treatments. It indicated that expression of genes was abundant and molecular activity was the most active in pathways that response to drought stress during this stage. In A vs C, the number of DEGs of Medicago ruthenica decreased significantly, the plant gradually adapted to the drought stress, and the molecular activity tended to be stable. To sum up, more genes were up-regulated in the leaves of the forage to respond to drought stress, while after rewatering, more genes were down regulated(Fig. 4). 
According to Venn analysis, there were 191 DEGs (93 up-regulated genes, 98 down-regulated genes) in $\mathrm{AB}$ vs $\mathrm{AC}$ (Additional file 1: Table S1; Fig. 5). There were 240 DEGs (50 up-regulated genes, 161 down-regulated genes) in $\mathrm{AB}$ vs $\mathrm{AD}$. There were 43 differential genes ( 8 up-regulated genes, 35 down-regulated genes) in $\mathrm{AB}$ vs $\mathrm{AC}$ vs $\mathrm{AD}$ (Additional file 2: Table S2; Fig. 5). Different treatments also had their own specific DEGs. There were 1563, 270 and 684 DEGs that only expressed in A vs B, A vs C and A vs D respectively (Fig. 5). The results showed that different measures were taken by the plant to respond to distinct external environment.

\section{Go enrichment analysis of DEGs under different treatment}

The Blast 2 GO v 2.5 software was used to analyze the GO enrichment of these DEGs in order to well known the biological functions of them. The results showed that 1,319 among 1,923 DEGs were annotated successfully in A vs B, including 987 up-regulated genes and 332 down-regulated genes. In the BP category, the top three enriched terms were "oxidation-reduction process" (126 up-regulated genes, 48 down-regulated genes), “carbohydrate metabolic process” (85 up-regulated genes, 47 down-regulated genes) and "development process" (126 up-regulated genes, 48 down-regulated genes). Most of the DEGs enriched in above biological processes were up-regulated. In addition, DEGs were also significantly enriched in antibiotic, polyol, inositol, glycoside biosynthetic and metabolic process, drug metabolic process and mitochondrial electron transport, cytochrome c to oxygen biological process and they were all up-regulated. Under the "cellular component" group, a lot of DEGs were classified into "respiratory chain complex IV", "respiratory chain", "cytochrome complex", and most of them were up-regulated. Their molecular functions were mainly related to "oxidoreductase activity", "hydrolase activity" and "electron carrier activity". The genes enriched in "oxidoreductase activity" and "electron carrier activity" 
were mostly up-regulated, while the genes enriched in "hydrolase activity" were mostly down-regulated (Additional file 3: Table S3; Fig. 6).

316 among 489 DEGs were successfully annotated in A vs C, including 132 up-regulated genes and 184 down-regulated genes. At this stage, DEGs were mainly involved in carbohydrate metabolism process (glucan, polysaccharide, sucrose, starch, disaccharide and oligosaccharide metabolic process), and most of the genes were down-regulated. In addition, the DEGs were also significantly enriched in cell wall related biological processes (cell periphery, cell wall organization/modification), and all of them were down-regulated. In the CC category, cell wall and external encapsulating structure were the dominant enriched terms. The molecular functions of these down-regulated DEGs were mainly related to enzyme activities, including pectinesterase activity, hydrolase activity, antioxidant activity, oxidoreductase activity and peroxidase activity etc.. The up-regulated genes of this process were mainly concentrated in inositol metabolic process and a series of catabolic processes, including cellular carbohydrate catabolic process, polyol catabolic process and inositol catabolic process, alcohol catabolic process and organic hydroxy compound catabolic process. And their molecular functions were mainly related to the enzyme activity, including inositol oxygenase activity, glutamate-5-semialdehyde dehydrogenase activity, and glutamate-5-kinase activity and amino acid kinase activity (Additional file 4: Table S4; Fig. 6).

632 among 924 DEGs were annotated successfully in A vs D, including 263 up-regulated genes and 369 down-regulated genes. The DEGs were mainly involved in single-organism metabolic process, carbohydrate metabolic process, polysaccharide metabolic process, polysaccharide and carbohydrate catabolism, glucan metabolic process, oligosaccharide metabolic process and 
disaccharide metabolic process. For the "molecular function" group, hydrolase activity, oxidoreductase activity, beta-amylase activity and dioxygenase activity were the most abundant subcategories. The molecular function of the up-regulated genes was mainly related to amylase activity, which were involved in the process of polysaccharide catabolic; the molecular function of the down-regulated genes was related to hydrolase activity, which were involved in carbohydrate metabolic process (Additional file 5: Table S5; Fig. 6).

According to the analysis of the enrichment of KEGG metabolic pathway, unigenes were assigned to 104 pathways and 20 pathways were significantly enriched ( $p$-value $<0.05$ ). Drought stress 9d, the top $10 \mathrm{KEGG}$ pathways with the highest representation of DEGs were: Protein processing in endoplasmic reticulum, Galactose metabolism, Starch and sucrose metabolism, Oxidative phosphorylation, Arginine and proline metabolism, TCA cycle, Phenylpropanoid biosynthesis, Pentose and glucuronate interconversions, Cysteine and methionine metabolism, Inositol phosphate metabolism. The up-regulated genes were mainly enriched in Protein processing in endoplasmic reticulum, Galactose metabolism, Oxidative phosphorylation, Starch and sucrose metabolism, Arginine and proline metabolism, TCA cycle, Glycolysis/Gluconeogenesis, Plant pathogen interaction, Cysteine and methionine metabolism, Inositol phosphate metabolism. The down-regulated genes were mainly enriched in Starch and sucrose metabolism, Photosynthesis-antenna proteins, Pentose and glucuronate interconversions, Circadian rhythm-plant, Porphyrin and chlorophyll metabolism, Nitrogen metabolism, Plant hormone signal transduction (Additional file 6: Table S6; Fig. 7).

Drought stress 12d, DEGs were mainly enriched in Pentose and gluconate conversions, Starch and sucrose metabolism, Arginine and proline metabolism, Photosynthesis-antenna proteins, 
Cysteine and methionine metabolism, Ascorbate and aldarate metabolism, Inositol phosphate metabolism, Nitrogen metabolism. The up-regulated genes were mainly enriched in Arginine and proline metabolism, Cysteine and methionine metabolism, Ascorbate and aldarate metabolism, Inositol phosphate metabolism,Valine, leucine and isoleucine biosynthesis and degradation, Carotenoid biosynthesis. The down-regulated genes were mainly enriched in Photosynthesis-antenna proteins, Pentose and gluconate conversions, starch and sucrose metabolism, Porphyrin and chlorophyll metabolism, Nitrogen metabolism, Galactose metabolism (Additional file 7: Table S7; Fig. 8).

During rehydration, the DEGs were mainly concentrated in Starch and sucrose metabolism, Flavonoid biosynthesis, Valine, leucine and isoleucine degradation, Circadian rhythms-plant. Most of the genes involved in Flavonoid biosynthesis, Circadian rhythms-plant, Vitamin B6 metabolism and Diterpene biosynthesis were up-regulated, while the genes involved in Starch and sucrose metabolism, Valine, leucine and isoleucine degradation, Plant hormone signal transduction were down-regulated (Additional file 8: Table S8; Fig. 9).

\section{Quantitative real-time PCR analysis}

In order to verify the accuracy of transcriptome sequencing, 6 DEGs were selected for qRT-PCR, including a glucan endo-1,3-beta-glucosidase (Fig. 10A), a ethylene response transcription factor ERF034 (Fig. 10B), a photosynthesis related gene carotene isomerase (Fig. 10C), a lipid metabolism related gene GDSL lipase (Fig. 10D), a DNA damage repair/tolerance protein DRT100 (Fig. 10E), and a fatty acid desaturase (Fig. 10F). The Medicago ruthenica MrActin gene was used as the endogenous reference. The expression patterns detected in qRT-PCR fit well with 
those in RNA-seq analysis (Fig. 10). Such results demonstrated that DEGs identified based on transcriptome sequencing were reliable.

\section{Disscussion}

In this study, we compared the differences of gene expression of Medicago ruthenica seedlings under drought stress $(9 \mathrm{~d}, 12 \mathrm{~d})$ and rehydration with normal water supply. 2,905 DEGs were screened out from massive transcriptome data. 1,923 (1411 up-regulated genes, 512 down-regulated genes), 489 (226 up-regulated genes, 263 down-regulated genes) and 924 (387 up-regulated genes, 537 down-regulated genes) genes were obtained respectively on the 9th day, 12th day of continuous drought stress and rehydration. It can be concluded that under moderate drought stress (9d), there were abundant genes expressed in the pathways of Medicago ruthenica, which positively responded to the stress by up-regulated. Under severe drought stress (12d), the number of DEGs decreased, indicated that the plant may gradually adapt to the drought stress, and the molecular activity tended to be stable. However, after rehydration, the number of DEGs increased again, the molecules were active, and most of them were down-regulated. After rehydration, only 240 genes were still significantly different among 2,221 genes that expressed under drought stress. The expression of the other genes basically returned to the normal level, which showed that the compensation effect of rehydration was remarkable, but it didn't mean $100 \%$ repair. Meanwhile, some new different genes were also produced.

According to venn analysis of the DEGs under different treatments, there were 191 DEGs in 9th vs 12 th day of drought stress. These genes may be helpful for Medicago ruthenica to cope with different degrees of drought stress, and can be used as candidate genes to improve the stress 
resistance of other plants. There were 43 DEGs shared by the 3 treatments, which may not only participate in the response of Medicago ruthenica to drought stress, but also joined in the process of restoration after rehydration. These genes can be used as the candidate genes to strengthen the drought adaptability of plants. Moreover, in addition to the common genes, there were also specific DEGs existed in each treatment. 1563, 270 and 684 DEGs were expressed specific on the 9th, 12th day of drought stress and rehydration respectively. The result showed that a great quantity of new DEGs appeared in different treatments, indicating that different strategies may be adopted to adapt the different degrees of drought stress and rehydration. Therefore, we analyzed the adaptive strategies of Medicago ruthenica in different treatment by GO and KEGG.

\section{Enrichment analysis of DEGs}

In this study, 2905 DEGs were screened out and their GO and KEGG enrichment analysis were carried out. The results also proved that Medicago ruthenica adopted distinct strategies to adapt the different degrees of drought stress and rehydration for a further step. After 9 days of drought stress, it was found that the molecular function of DEGs was mainly related to the activities of oxidoreductase, hydrolase and electron transport, and they were mainly enriched in the process of oxidation-reduction, carbohydrate metabolism and development. Most of the genes were up-regulated, which had a positive effect on the drought tolerance of Medicago ruthenica. It noted that Medicago ruthenica mainly induced the expression of genes that related to oxidoreductase and hydrolase to adjusted the oxidation-reduction, carbohydrate metabolism and development in plants. This was its answer to moderate stress.

Stress 12 days, the molecular function of the up-regulated genes was mainly related to enzyme 
activity. They involved in inositol metabolism and a series of catabolism processes, including carbohydrate catabolism, polyol catabolism, inositol catabolism, alcohol catabolism and organic hydroxyl compound catabolism. It was suggested that Medicago ruthenica could cope with the severe drought stress (12d) by inducing up-regulation of genes involved in catabolism which could promoted the decomposition of organic matter. Most of the genes involved in carbohydrate metabolism processes (including Intracellular carbohydrate metabolism, Polysaccharide metabolism, Glucan metabolism, Starch and sucrose metabolism, Disaccharide metabolism and Oligosaccharide metabolism) were down-regulated and connected with the activities of pectinase, antioxidant, oxidoreductase and peroxidase. It revealed that the carbohydrate metabolism of Medicago ruthenica was strongly inhibited by severe drought stress, and the antioxidant defense system was also weakened. To sum up, under severe drought stress, on the one hand, Medicago ruthenica reduced the material and energy consumption of the body for a further step by inhibit the carbohydrates metabolism process. On the other hand, it promoted the further decomposition of other organic matters stored in the body to adapt to severe drought stress.

During rehydration, the DEGs were mainly involved in carbohydrate metabolism, polysaccharide metabolism, glucan, oligosaccharide, disaccharide metabolism processes. The molecular function of the up-regulated genes was mainly connected with the activity of amylase and involved in the process of polysaccharide catabolism. The molecular function of the down-regulated genes was related to the activity of hydrolase and participated in carbohydrate metabolism process. At this time, the inhibition of carbohydrate metabolism had not been released. Medicago ruthenica still used the soluble substances produced in the process of polysaccharide catabolism as the sources of energy, carbon and nitrogen. It was convenient for centralized supply 
of nutrients after rehydration. On the one hand, it was conducive to accelerating the plant recovery process[14]. On the other hand, it played a role of osmotic regulation to strengthen the ability of absorbing water and was helpful to the over recovery of leaf water potential and water transport efficiency.

Under drought stress, plants usually start a complex network regulation mechanism to change the genes' expression level, promote the interaction between biochemical and molecular processes in the body, so as to adapt to the stress environment of water deficiency. In this study, the DEGs and its concentrated metabolic pathways were screened out by transcriptome analysis. It reflected the regulatory process of Medicago ruthenica's response to sustainable drought stress and rehydration on the transcriptional level. It was helpful to preliminarily explained the molecular mechanism of adaptation to drought stress and rehydration.

To analyse the significantly enriched metabolic pathways under different treatments. It was found that most of the DEGs enriched in Galactose metabolism, Arginine and proline metabolism, TCA cycle, Inositol phosphate metabolism, Glycolysis/gluconeogenesis, Starch and sucrose metabolism, Plant-pathogen interaction, Cysteine and methionine metabolism were up-regulated in response to drought. However, the genes enriched in Photosynthesis-antenna proteins, Porphyrin and chlorophyll metabolism, Nitrogen metabolism, Circadian rhythm-plant and Plant hormone signal transduction were mostly down-regulated to respond to the adversity. The regulation of the above metabolic pathways may be beneficial to the adaptation of Medicago ruthenica to moderate drought stress (9d).

In this period, Medicago ruthenica mainly up regulating the DEGs in "Galactose metabolism", 
"Arginine and proline metabolism" and "Starch and sucrose metabolism" to play a role in osmoregulation. In this work, $\alpha$-galactosidase, GOLS, HK and PGM were up-regulated in "Galactose metabolism". Galactose, as a soluble sugar, played an important role in osmoregulation. The change of gene expression in this pathway could enhance the water holding capacity and improve the drought tolerance of Medicago ruthenica. These genes were in favor of the active adaptation of the forage to arid environment. Simultaneously, 11 P5CS and a OAT were up-regulated in "Arginine and proline metabolism". Proline, as a major osmotic protective substance, was synthesized a lot in arid environment. Its synthesis was connected with the temporary starch degradation, and played a role in plant to cope with drought stress[15]. Proline was the most soluble amino acid with strong hydrophilicity. And it could make for ammonia detoxification and protection of plasma membrane. It was found that there were two pathways for proline synthesis in plants: P5CS and OAT[16,17]. In our study, only one OAT gene was identified in the whole process of drought stress, indicating that the plant mainly synthesized proline through P5CS pathway. So, Medicago ruthenica synthesized a large amount of proline by inducing the up-regulation of P5CS in a water shortage environment. It can not only stabilize the protoplast colloid and metabolism process in tissues, help cells and tissues to retain water, reduce water loss in plants, but also protect the integrity of plasma membrane by detoxification. Besides, $\beta$-fructofuranosidase genes, $\beta$-glucosidase genes, galacturonidase genes, galU, GPI, HK and PGM in "Starch and sucrose metabolism" were up-regulated in this period. These genes promoted the decomposition of non-structural carbohydrates, produced soluble sugar, protein and released energy when plants suffered from water deficit. The expression of these genes not only played the role of osmotic regulation but also provided energy for plants to resist drought. We also found a 
up-regulated gene TPS in this pathway. It was a key enzyme gene in trehalose synthesis pathway, and trehalose was related to the tolerance of plants to abiotic stress. Yang[18] found that the length, diameter, number of primary and secondary lateral roots, total root area and root activity of transgenic maize containing TPS1 increased obviously. The expression of this gene might promote the root growth and increase the root/shoot ratio of Medicago ruthenica, which was beneficial to withstand the moderate drought $(9 \mathrm{~d})$.

During this stage, "Nitrogen metabolism" was inhibited by the down-regulated genes NR and GLT1 and the pathways related to carbohydrate metabolism was promoted in Medicago ruthenica. It was suggested that the Oxalacetate-Malate Shuttle, which is responsible for the conversion of NADPH produced by light reaction to NADH in cytoplasm, may be a valve on chloroplast membrane. The closed valve inhibited the export of reducing products in chloroplast Malic acid accumulated, $\mathrm{CO}_{2}$ assimilation was blocked and $\mathrm{NO}_{2}{ }^{-}$reduction was promoted. Conversely, it was conducive to the assimilation of $\mathrm{CO}_{2}$ and photosynthesis of plants[19]. Therefore, inhibiting Nitrogen metabolism and using more energy to promote carbohydrate metabolism may be one of the mechanisms of Medicago ruthenica to adapt to drought stress and rehydration.

Under the moderate drought stress (9d), the antioxidant defense system was also activated in response to drought stress. In the process of plant growth, drought stress induced plant oxidative stress and produced a lot of ROS. Low concentration of ROS could act as a second messenger to mediate multiple responses in cell signaling pathways, but high concentration of ROS could cause cell damage or even death[20,21]. In order to against ROS toxicity, Medicago ruthenica activated its antioxidant system to maintain or reconstruct redox balance. The excessive ROS in the body was eliminated through various metabolic pathways including Peroxisome, Glutathione 
metabolism[22]. 3 genes encoding SOD in "Peroxisome" were up-regulated under drought stress. 2 of them annotated to $\mathrm{Cu}-\mathrm{Zn}$ superoxide dismutase family and another one annotated to Fe- $\mathrm{Mn}$ superoxide dismutase family. Therefore, Medicago ruthenica regulated the activity of corresponding enzymes by up regulating the antioxidant enzyme genes. The plant positively responded to moderate drought stress in this way. Meanwhile, 2 GST genes and a GSR gene in "Glutathione metabolism" were all up-regulated to participate in the response to drought. GST participated in the defense of plants to various stresses by eliminating the increased endogenous phytotoxins under oxidative stress[23]. Generally speaking, SOD1, SOD2, GST, GSR were all involved in the antioxidant defense of Medicago ruthenica through up-regulation. The obvious change of antioxidant activity accelerates the conversion of $\mathrm{O}_{2}{ }^{-}$to $\mathrm{H}_{2} \mathrm{O}_{2}, \mathrm{H}_{2} \mathrm{O}_{2}$ to $\mathrm{H}_{2} \mathrm{O}$ and $\mathrm{O}_{2}$, effectively alleviates the peroxide damage caused by drought, which made the plant showed a strong ability to scavenging ROS. Through these pathways, Medicago ruthenica could reestablish a new equilibrium and maintain a lower ROS concentration, which could prevent tissue and cells from dying due to oxidative stress damage[24].

Moreover, Medicago ruthenica also could resist the stress of this stage by slowing down its growth. In our study, photosynthesis of the forage was inhibited under the continuous drought stress, and many related genes were significantly down regulated. This result were similar to those of De[25], Hanf[26] and Behringer[27]. In the forage, a total of 10 LHC genes, including 7 LHCB1 genes and 3 LHCB3 genes, were identified in "Photosynthesis-antenna proteins" under this condition. And all of them were down-regulated. Consequently, the light harvesting ability of Medicago ruthenica was obviously limited and its photosynthesis was suppressed. So, the decrease of photosynthetic capacity of the plant under water deficit was mainly caused by the 
decrease of light harvesting capacity. The inhibition of "Nitrogen metabolism" also affected the synthesis of chlorophyll to a certain extent. The NADPH-protochlorophyllide oxidoreductase gene in "Porphyrin and chlorophyll metabolism" was down-regulated, which inhibited the production of chlorophyllide. Although the photosynthetic capacity of the plant was inhibited, "Carbon fixation in photosynthetic organisms" was still in progress. There were two MDH, two PGK, a phosphoenolpyruvate carboxykinase and a GAPDH enriched in this pathway and all of them were up-regulated. That is, the photosynthesis of Medicago ruthenica was still in progress so that the plants could grow slowly and the biomass could increase continuously. At the same time, under drought stress, stomata tend to be closed, and the $\mathrm{CO}_{2}$ absorbed from the atmosphere was reduced. It caused extensive accumulation of NADPH. The synthesis of proline consumed NADPH and ATP and provided $\mathrm{NADP}^{+}$and ADP for photosynthesis of plants. It stimulated the photosynthesis of plant so that Medicago ruthenica could maintain a certain growth rate.

To maintain the above metabolism, the body needs enough energy supply. Under moderate drought stress, energy supply in Medicago ruthenica mainly depends on "TCA cycle" and "Pentose phosphate". Genes in "TCA cycle" were up-regulated, including ACO, IDH, MDH and so on. Meanwhile, genes in "Pentose phosphate", including PGD and PGM, were also up-regulated to generate ATP. It provided energy for the life activities of Medicago ruthenica. Additionally, INO1, MIP synthase related gene in "Inositol phosphate metabolism", was up-regulated to synthesize myo-inositol. Under the action of TPI, it produced glyceraldehyde-3P and entered the "Glycolysis/gluconeogenesis", which could also provide energy for the plant.

After 12 days of drought stress, most of the genes enriched in "Arginine and proline metabolism", "Cysteine and methionine metabolism", “Ascorbate and aldarate metabolism", 
"Inositol phosphate metabolism", "Valine, leucine and isoleucine degradation", "Carotenoid biosynthesis" were up-regulated, while those enriched in "Photosynthesis-antenna proteins", "Starch and sucrose metabolism", "Galactose metabolism", "Nitrogen metabolism", "Porphyrin and chlorophyll metabolism", and "TCA cycle", were down-regulated in response to severe drought stress. It proved that serious drought stress suppressed the metabolism of carbohydrate and photosynthesis but promoted the metabolism of amino acid.

LHCB1, LHCB2, LHCB5, LHCA4 and rbcS in "Photosynthesis-antenna proteins" were significantly down-regulated. The wilting and curling of leaves reduced light absorption, HEMC/HMB and $\mathrm{ChlH}$ were down regulated in "Porphyrin and chlorophyll metabolism", it affected the synthesis of chlorophyll-a. Thus, photosynthesis was further inhibited. Additionally, ATP synthesis and sugar metabolism were much weakened, membrane structure was destroyed, MDA increased suddenly. The metabolism of the body was disordered, and the cell vitality and repair ability were obviously attenuated. Under severe drought stress, the effect of antioxidant enzymes was gradually weakened. Medicago ruthenica synthesized the antioxidant to scavenge reactive oxygen species and protect membrane structure. The up regulation of Z-ISO gene in "Carotenoid biosynthesis" promoted carotenoid synthesis, it was benefit to quench singlet oxygen and resist injury. Chlorophyll decomposes in the aging process of old leaves, which made the original carotenoids exposed and resulting in the etiolation of leaves. This is a process of recovering nutrients from old tissues[28]. At the same time, myo-inositol was oxidized to produce D-glucuronate and AsA under the action of up-regulated gene MIOX. Synthesized AsA through the inositol pathway of ascorbic acid biosynthesis, Medicago ruthenica could scavenge reactive oxygen species, inhibit membrane lipid peroxidation, stabilize cell membrane structure and delay 
cell senescence. It can also synthesized cell wall polysaccharide, pectin and xylan, formed cellulose to strengthen cell wall and maintain cell wall shape.

The down-regulation of pectinase and starch phosphorylase genes in "Starch and sucrose metabolism" inhibited the degradation of cell wall and maintained the shape of it. Moreover, the genes related to amino acid metabolism process were obviously up regulated. Medicago ruthenica significantly up regulating P5CS in "Arginine and proline metabolism". The plant continued to synthesize proline to relieve osmotic pressure to resist drought stress. The genes enriched in "Cysteine and methionine metabolism" were up-regulated. L-methionine produced S-adenosyl-L-methionine under the action of metK, then produced 1-Aminocyclopropane-1-carboxylate under the action of ACS, and finally produced ethylene. It might cause the old leaves to fall off. This finally cut down the biomass. In order to maintain energy supply, "Valine, leucine and isoleucine degradation" was activated under severe drought stress. Succinyl CoA and Acetyl CoA were produced by BCAT2 and MCCA in this pathway, which entered the TCA cycle and provide energy for physiological metabolism.

In conclusion, under severe drought stress, photosynthesis of Medicago ruthenica decreased gradually, and its products decreased accordingly. The forage mainly relied on the synthesis of proline and the decomposition of other stored organic matter to carry out osmotic adjustment, obtain energy and sacrifice part of biomass to maintain physiological metabolism. This was a passive response to drought stress. Apart from that, Medicago ruthenica also synthesized two antioxidants, carotenoids and ascorbic acid, to alleviate oxidative damage and adapt to drought stress. Ultimately, Medicago ruthenica adapted to the drought stress by sacrificing its own biomass. 
After rehydration, most of the genes enriched in "Flavonoid biosynthesis", "Circadian rhythm-plant", "Vitamin B6 metabolism", "Carotenoid biosynthesis" and "Diterpene biosynthesis" were up-regulated to participate in the rehydration recovery process of Medicago ruthenica. "Photosynthetic-antenna protein" was restored and the limitation of light capture ability of Medicago ruthenica was relieved. However, "Carbon fixation of photosynthetic tissue" was not completely restored. "Glycerolipid metabolism" was significantly enriched under the condition of rehydration, and the expression of GPAT in it was up-regulated. GPAT was the first enzyme in the pathway of glycerin synthesis, which was helpful for the repair of photosynthetic membrane system and the recovery of photosynthesis. Carbonic anhydrase $\beta$-CA5 in N metabolism was significantly down-regulated after rehydration, which maintained the relative stability of photosynthetic rate to a certain extent.

Besides, "Starch and sucrose metabolism" was also not completely recovered. Medicago ruthenica still relied on the decomposition of other nutrients to repair its damage. At this moment, HMGL and hydroxymethylglutaryl CoA synthetase in "Valine, leucine and isoleucine degradation" were up-regulated to generate acetyl coenzyme A. It may be involved in "TCA cycle", "Tryptophan skeleton biosynthesis", "Ketone synthesis and degradation", and could provide $\mathrm{C}, \mathrm{N}$ and energy for the repair of the herbage. Proline had a unique structure, and could be used as a molecular chaperone to protect the integrity of protein and the activity of enzymes. When drought stress was relieved, proline in plants could be oxidized and decomposed. This process generates large amounts of FADH2 and NADPH and released a large amount of free energy to generate ATP. It provided the necessary energy for the restoration of plants after stress.

After rehydration, many significantly enriched metabolic pathways under drought stress were 
recovered, but there were still some metabolic pathways significantly enriched. It is speculated that these metabolic pathways may be beneficial to the recovery process of Medicago ruthenica. "Flavonoid biosynthetic" was significantly enriched under this treatment. In this pathway, 4 CHS, 5 Flavanone 3-hydroxylase, 2 flavonoid hydroxylase and a caffeinyl CoA O-methyltransferase genes were all up-regulated. Flavonoid was secondary metabolites in higher plants. It was important antioxidants in plants and had multiple functions. Through increasing the activity of peroxidase and reducing the content of MDA, it could eliminate and reduce the damage of active oxygen caused by drought. It could also indirectly enhance the drought resistance of plants by increasing abscisic acid. Therefore, the synthesis of flavonoids in plants played an important role in improving the drought resistance of plants. CHS was the key gene in this pathway, and its up-regulation promoted the synthesis of flavonoids. This was conducive to repaired oxidative damage under severe drought stress. Medicago ruthenica could recover quickly by regulating the synthesis of flavonoids and changing the proportion of flavonoids. At the same time, after rehydration, Z-ISO in "Carotenoid biosynthesis" was up-regulated, which promoted the formation of lycopene. Some of them synthesized $\alpha$-carotene and then produced lutein under catalysis of $\beta$-carotene hydroxylase. The other part formed $\beta$-carotene. Under the action of $\beta$-carotene hydroxylase gene, some of them produced zeaxanthin, viola yellow and neoxanthin. Under the action of 9-cis-epoxy carotenoid dioxygenase gene NCED, they participate in ABA biosynthesis. And the other part of $\beta$-carotene synthesized astaxanthin. Lutein and astaxanthin are antioxidants with strong antioxidant capacity. It not only can remove nitrogen dioxide, sulfide, disulfide and so on. It can also effectively quench the highly oxidized singlet reactive oxygen species and other free radicals in the environment. And achieved the goal of reducing lipid peroxidation, stabilizing 
membrane structure and protecting important molecules from oxidative damage. After the restoration of water condition, a large number of flavonoids, carotenoids and astaxanthin were synthesized in Medicago ruthenica to remove the accumulated reactive oxygen species and repair the oxidative damage under water stress.

In addition, "Circadian rhythm-plant" was also remarkable enriched in the recovery process of Medicago ruthenica. Under abiotic stress, plants regulated the optimal time of important physiological activities by changing circadian rhythm. This improved the adaptability of plants to adversity[29]. At present, many studies had confirmed that the circadian rhythms of plants were directly related to the response of plants to drought stress[30]. Some critical drought genes and physiological activities induced by drought showed differences between day and night[31], and could regulate the opening and closing of stomata. However, in this study, although the pathway was enriched both in drought stress and rehydration, it was more significantly enriched in the process of rehydration. It was suggested that rehydration had a significant effect on the circadian rhythm of Medicago ruthenica, and the regulation of the pathway may be beneficial to the recovery of its growth. This pathway including up-regulated genes FKF1, PRR5, TOC1 and down-regulated gene LHY. These genes may be involved in the light signal network transmission. Regulating the circadian rhythm of plants through these differential genes may accelerate the recovery process of Medicago ruthenica. It was speculated that these genes may be the key genes to regulated the circadian rhythm in response to rehydration. The up or down regulation of these genes affected the photosynthetic pathway, flowering, and cell elongation of euphorbiella sinensis. Moreover, CHS in "Flavonoid biosynthesis" was also involved in this pathway, and its up-regulation may be beneficial to UV-B protection of leaves. 
These biological functions and metabolic pathways, which were significantly enriched, may be played a key role in Medicago ruthenicus in response to drought and rehydration. The enriched DEGs may be participated in the response of Medicago ruthenicus to drought stress and rehydration, and had a positive effect on the drought tolerance and rehydration recovery of the herbage. The results provide a data and scientific basis for further revealing the molecular mechanism of Medicago ruthenicus adaptation to drought and rehydration.

\section{Conclusion}

This research found the critical pathway of Medicago ruthenica in response to drought and rehydration. And on this basis, we screened out the genes related to drought resistance for further, did a preliminary analysis about the stage response and adaptive strategy of the plant to different degree of drought stress and rehydration.

Under moderate drought stress (9d), Medicago ruthenica increased osmoregulation substance and maintained osmolarity mainly by promoting carbohydrate metabolism (Galactose metabolism, Starch and sucrose metabolism), amino acid metabolism (Arginine and proline metabolism). And it was conducive to improve the osmoregulation ability. Meanwhile, the plant also inhibited photosynthesis related metabolic pathways (Photosynthetic-antenna protein, Porphyrin and chlorophyll metabolism) to reduced light absorption and chlorophyll synthesis. It slowed down the plant growth. They were the 2 major ways taken by Medicago ruthenica to positively adapt to adversity.

Under severe drought stress, the carbohydrate metabolism and TCA cycle of Medicago 
ruthenica were weakened, and the photosynthesis related pathways were still inhibited. The forage mainly relied on the synthesis of proline (Arginine and proline metabolism) and the decomposition of other stored organic matter (Valine, leucine and isoleucine degradation) to carry out osmotic adjustment, obtain energy and sacrifice part of biomass to maintain physiological metabolism concurrently. This was a passive response to drought stress. Additionally, Medicago ruthenica synthesized AsA through Ascorbate and aldarate metabolism, which was benefit to alleviate oxidative damage and protect membrane structure. It was contributed to survival in adversity.

After rehydration, the physiological metabolism of Medicago ruthenica has not been completely recovered. It not only promoted carbohydrate hydrolysis to produce D-glucose and maltose (Starch and sucrose metabolism), but also relied on the decomposition of other nutrients (Valine, leucine and isoleucine degradation) to provide $\mathrm{C}, \mathrm{N}$ and energy and enhance the water uptake ability. After the restoration of water condition, a large number of flavonoids, carotenoids and astaxanthin (Flavonoid biosynthesis, Carotenoid biosynthesis) were synthesized in Medicago ruthenica to remove the accumulated reactive oxygen species and repair the oxidative damage under water stress. Except for above 3 ways, the regulation of Circadian rhythm-plant may accelerate the recovery process. Our transcriptome data dramatically enriches the resource of resistance gene. A new idea was put forward to enhance the drought resistance of alfalfa and other plants. It also laid a foundation for drought-resistant breeding of herbage.

\section{Methods}




\section{Sample collection and preparation}

Medicago ruthenica $(\mathrm{L}.) \mathrm{cv}$. Zhilixing was selected as the experimental material. Scholars from Inner Mongolia Agricultural University cultivated this variety by multiple mixed selection method and then registered as bred variety. It is a perennial herb with erect stem, more branches( $30 \sim 50$ per plant), larger and abundant leaves. It is an early-maturing variety has good palatability and high feeding value. The yield of fresh grass per hectare is $37500 \sim 45000 \mathrm{~kg}$. The yield of seed is $150-180 \mathrm{~kg} / \mathrm{ha}$, which is $50.6 \%$ higher than that of the original seed. The suitable harvest time is the late yellow ripening stage. Meanwhile, the hard seed rate decreased from $60 \% \sim 30 \%$ to about $10 \%$. Its green period was from 180 to 200 days. This variety has strong resistance to adversity and is suitable to cultivate in arid and cold areas.

Seeds of this variety were collected in September 2008 from experimental fields of Inner Mongolia Agricultural University located in Hohhot, Inner Mongolia, China. Select plump seeds for experiment. All seeds were soaked in concentrated $\mathrm{H}_{2} \mathrm{SO}_{4}$ for 5-8 min in order to break the hard seed of Medicago ruthenica (L.) cv. Zhilixing. And then Germination at constant temperature of $20{ }^{\circ} \mathrm{C}$. After the seeds germinated, transferred them to float tray and placed them in the greenhouse with $12 \mathrm{~h}$ light per day, regular and quantitative water supply until the seedlings grow 6-8 leaves. The seedlings need sufficient water before continuous drought stress was carried out.

The experiment was divided in 2 groups: Control group (CK) maintained normal watering. The treatment group was not watered. After 12 days of continuous water control treatment, the leaves of seedlings wilted, turned yellow and fell off, and then began to rehydrate with sufficient moisture. After 4 days rehydration, the plants returned to normal growth. There were 3 replicates 
in both the CK group and the treatment group. Samples were collected from different treatments including CK, drought stress (9d, 12d) and rehydration (4d). There were 4 treatments and 12 samples in total, and the fourth or fifth mature leaf was selected for each treatment. All samples were immediately frozen in liquid nitrogen and stored at $-80{ }^{\circ} \mathrm{C}$ for RNA extraction.

\section{RNA extraction and transcriptome sequencing}

RNA degradation and contamination was monitored on $1 \%$ agarose gels. RNA purity was checked using the NanoPhotometer ${ }^{\circledR}$ spectrophotometer (IMPLEN, CA, USA). RNA concentration was measured using Qubit ${ }^{\circledR}$ RNA Assay Kit in Qubit ${ }^{\circledR}$ 2.0 Flurometer (Life Technologies, CA, USA). RNA integrity was assessed using the RNA Nano 6000 Assay Kit of the Agilent Bioanalyzer 2100 system (Agilent Technologies, CA, USA).

The total RNA of each sample was $1.5 \mu \mathrm{g}$. Sequencing libraries were generated using NEBNext ${ }^{\circledR}$ Ultra $^{\text {TM }}$ RNA Library Prep Kit for Illumina ${ }^{\circledR}$ (NEB, USA). The library fragments were purified with AMPure XP system(Beckman Coulter, Beverly, USA) to select cDNA fragments of $250 \sim 300 \mathrm{bp}$ in length. Then $3 \mu$ l USER Enzyme(NEB, USA) was used with size-selected, adaptor-ligated cDNA at $37^{\circ} \mathrm{C}$ for $15 \mathrm{~min}$ followed by $5 \mathrm{~min}$ at $95{ }^{\circ} \mathrm{C}$ before PCR. Then, to perform PCR with Phusion High-Fidelity DNA polymerase, Universal PCR primers and Index(X) Primer. Finally, PCR products were purified(AMPure XP system) and library quality was assessed (Agilent Bioanalyzer 2100 system).

\section{Sequence assembly and Unigene annotation}

Transcriptome assembly was accomplished based on the left.fq and right.fq using Trinity[32] with min_kmer_cov set to 2 by default. All other parameters also set default. Gene function was 
annotated based on the following databases: NR, NT, Pfam, KOG/COG, Swiss-Prot, KO, GO.

\section{Analysis of DEGs}

Using RSEM to estimate gene expression levels[33]. Using the DESeq R package(1.10.1) to perform the differential expression analysis of different groups. Genes with an adjusted P-value $<0.05$ found by DESeq were identified as DEGs. GO enrichment analysis of the DEGs was implemented by the GOseq $\mathrm{R}$ packages based Wallenius non-central hyper-geometric distribution[34]. KEGG[35] is used for understanding high-level functions and utilities of the biological system from molecular-level information (http://www.genome.jp/kegg/). We used KOBAS[36] software to test the statistical enrichment of DEGs in KEGG pathways.

\section{Real-time quantitative PCR analysis}

qRT-PCR analysis was performed to validate the DEGs identified by transcriptome sequencing. Total RNA were extracted as described before. The qRT-PCR assays were performed using Fast Super EvaGreen ${ }^{\circledR}$ qPCR Master Mix (US Everbright, Suzhou, China) in an ABI 7500 system (Applied Biosystems, USA) with the following program: $95{ }^{\circ} \mathrm{C}$ for $2 \mathrm{~min} ; 45$ cycles of $95{ }^{\circ} \mathrm{C}$ for $10 \mathrm{~s}, 55^{\circ} \mathrm{C}$ for $10 \mathrm{~s}, 72{ }^{\circ} \mathrm{C}$ for $30 \mathrm{~s}, 95^{\circ} \mathrm{C}$ for $15 \mathrm{~s}$ and $60{ }^{\circ} \mathrm{C}$ for $60 \mathrm{~s}$. A commonly used reference gene Actin was used to normalize the expression levels of target genes. The Medicago ruthenica Actin gene was selected as the endogenous reference. All the primers were synthesized by Invitrogen(Beijing, China) (Table 2). A $20 \mu 1$ reaction mix was set up containing $10 \mu 12 \times$ Fast

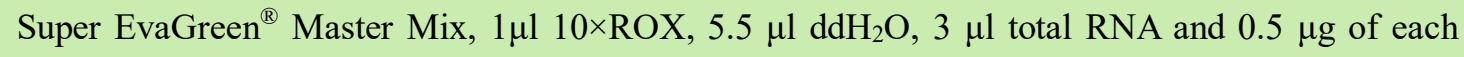
primer. The relative expression changes of the endogenous reference and tested genes were analyzed by the $2^{-\square \square \mathrm{CT}}$ method[37]. 


\title{
Additional files
}

Additional file 1: Table S1. DEGs in AB vs AC.(XLS $98.9 \mathrm{~kb})$

Additional file 2: Table S2. DEGs in AB vs AC vs AD.(XLS $22.1 \mathrm{~kb}$ )

Additional file 3: Table S3. GO functional annotations and the number of DEGs statistics in AB.(XLS $1250 \mathrm{~kb})$

Additional file 4: Table S4. GO functional annotations and the number of DEGs statistics in AC.(XLS $363 \mathrm{~kb})$

Additional file 5: Table S5. GO functional annotations and the number of DEGs statistics in AD.(XLS $638 \mathrm{~kb})$

Additional file 6: Table S6. Top 20 KEGG pathways of DEGs in drought stress AB.(XLS $1.24 \mathrm{~kb})$

Additional file 7: Table S7. Top 20 KEGG pathways of DEGs in drought stress AC.(XLS $1.34 \mathrm{~kb})$ Additional file 8: Table S8. Top 20 KEGG pathways of DEGs in AD.(XLS $1.34 \mathrm{~kb}$ )

\begin{abstract}
Abbreviations
BLAST: Basic Local Alignment Search Tool; DEGs: Differentially expressed genes; RT-qPCR: Real-time quantitative PCR; Nr: NCBI non-redundant protein sequences; Nt: NCBI non-redundant nucleotide sequences; Pfam: Protein family; KOG/COG: Clusters of Orthologous Groups of proteins; Swiss-Prot: A manually annotated and reviewed protein sequence database; GO: Gene Ontology; KO: KEGG Ortholog database; NCED: 9-cis-epoxycarotenoid dioxygenase; NR: Nitrate reductase; GLT1: Glutamate synthase; GOLS: Galactinol synthase; HK: hexokinase; PGM: phosphoglucomutase; P5CS: delta-1-pyrroline-5-carboxylate synthetase; OAT: ornithine--oxo-acid transaminase; galU: UTP--glucose-1-phosphate uridylyltransferase; GPI: glucose-6-phosphate isomerase; TPS: Trehalose-6-phosphate synthase; SOD: superoxide dismutase; GST: glutathione S-transferase; GSR: glutathione reductase; LHC:light-harvesting complex II chlorophyll a/b binding protein; MDH: malate dehydrogenase; PGK: Phosphoglycerate kinase; GAPDH: glyceraldehyde 3-phosphate dehydrogenase; ACO: aconitate hydratase; IDH: isocitrate dehydrogenase; PGD:6-phosphogluconate dehydrogenase; PGM:phosphoglucomutase; INO1: myo-inositol-1-phosphate synthase; MIP: Myo-inositol-1-phosphate synthase; HEMC/HMB: hydroxymethylbilane synthase; ChlH: magnesium chelatase subunit H; Z-ISO: zeta-carotene isomerase; MIOX: myo-inositol oxygenase; metK: S-adenosylmethionine synthetase; ACS: 1-aminocyclopropane-1-carboxylate synthase; CHS: chalcone synthase; FKF1: flavin-binding kelch repeat F-box protein 1; PRR5: pseudo-response regulator 5; TOC1: pseudo-response regulator 1; LHY: MYB-related transcription factor LHY; rbcS: ribulose-bisphosphate carboxylase small chain
\end{abstract}

\section{Declarations}

Ethics approval and consent to participate 
Not applicable.

\section{Consent for publication}

Not applicable.

\section{Availability of data and materials}

Datasets are available at NCBI project PRJNA655825. The transcriptome datasets are available in the NCBI Short Read Archive (SRA) database with the accession number: SAMN15756116, SAMN15756117, SAMN15756118, SAMN15756119, SAMN15756120, SAMN15756121, SAMN15756122, SAMN15756123, SAMN15756124, SAMN15756125, SAMN15756126, SAMN15756127.

\section{Competing interests}

The authors declare that they have no competing interests.

\section{Funding}

This study was supported by the National Natural Science Foundation of China (31460626), Science and technology project of Inner Mongolia Autonomous Region in China (2019GG244), Science and technology project of Inner Mongolia Agricultural University in China (YZGC2017008). The funding body had no role in the design of the study, collection, analysis, or interpretation of data or in the writing of the manuscript.

\section{Authors' contributions}

FLS and RNW conceived and designed the experiment. RNW performed the experiments, analyzed the data and wrote the manuscript. BX collected the plant materials. All authors reviewed and approved the final manuscript.

\section{Acknowledgements}

We thank Novogene Bioinformatics Technology Co.,Ltd (Beijing, China) for its technical support. This work was supported by the National Natural Science Foundation of China (31460626), Science and technology project of Inner Mongolia Autonomous Region in China (2019GG244), Science and technology project of Inner Mongolia Agricultural University in China (YZGC2017008).

[1] Xu ZZ, Zhou GS, Shimizu H. Plant response to drought and rewatering. Plant Singaling \&Behavior. 2010;(05):649-654. 
[2] Chen D, Wang S, Cao B, et al. Genotypic variation in growth and physiological response to drought stress and re-watering reveals the critical role of recovery in drought adaptation in Maize seedlings. Frontiers in Plant Science. 2015; 6:1241.

[3] Yang JY, Zheng W, Tian Y, et al. Effects of various mixed salt-alkaline stresses on growth, photosynthesis, and photosynthetic pigment concentrations of Medicago ruthenica seedlings. Photosynthetica. 2011; 49:275-284.

[4] Guan B, Zhou D, Zhang H, et al. Germination responses of Medicago ruthenica seeds to salinity, alkalinity, and temperature. Journal of Arid Environments. 2009;73:135-138.

[5] Li HY, Li ZY, Shi WG, et al. Leaf anatomic indexes and the relations with drought resistance of the six forage of Leguminosae. Acta Botanica Boreali-Occidentalia Sinica. 2010;30(10): 1989-1994.

[6] An SS, Darboux F, Cheng M. Revegetation as an efficient means of increasing soil aggregate stability on the Loess Plateau (China). Geoderma. 2013;209(1):75-85.

[7] Zhao LP, Wu GL, Shi ZH. Post-fire species recruitment in a semiarid perennial steppe on the Loess Plateau. Australian Journal of Botany. 2013;61(1):29.

[8] Campbell TA, Bao G, Xia ZL. Agronomic evaluation of Medicago ruthenica collected in Inner Mongolia. Crop Sci. 1997;37:599-604.

[9] Campbell TA, Bao G, Xia ZL. Completion of the agronomic evaluations of Medicago ruthenica[(L.) Ledebour] germplasm collected in Inner Mongolia. Genetic Resources and Crop Evolution. 1999;46:477-484.

[10] Zhang YT, Shi FL, Qiao Y, et al. Effects of EMS mutated Medicago ruthenica (L.) Sojak.cv.Zhilixing seeds on seedling emergence and seedling growth during germination stage. Grassland and Prataculture. 2019;31(02):24-31.

[11] Shi FL, Guo XX, Li H. Examination and analysis of drought-resisting morphology and anatomy of Melilotoides ruthenica. Agricultural Research in the Arid Areas. 2005;(02):115-118+233

[12] Palovaara J, Saiga S, Weijers D. Transcriptomics approaches in the early Arabidopsis embryo[J]. Trends in Plant Science, 2013, 18(9): 514-521

[13] Shu YJ, Li W, Zhao JY, Liu Y, Guo CH. Transcriptome sequencing and expression profiling of genes involved in the response to abiotic stress in Medicago ruthenica. Genetics \& Molecular Biology. 2018;41(3):638-648.

[14] An YY, Liang ZS, Zhao RK, et al. Organ-dependent responses of Periploca sepium to repeated dehydration and rehydration. South African Journal of Botany. 2011;77:446-454. 
[15] Zanella M, Borghi GL, Pirone C, Thalmann M, Pazmino D, Costa A, Santelia D, Trost P, Sparla F. $\beta$-amylase 1(BAM1) degrades transitory starch to sustain proline biosynthesis during drought stress. J Exp Bot. 2016;67:1819-1826.

[16] Xu ZY, Kim SY, Hyeon do Y, Kim DH, Dong T, Park Y, Jin JB, Joo SH, Kim SK, Hong JC, Hwang D, Hwang I. The Arabidopsis NAC transcription factor ANAC096 cooperates with b ZIP-type transcription factors in dehydration and osmotic stress responses. Plant Cell. 2013; 25:4708-4724.

[17] Szabados L, Savoure A. Proline: a multifunctional amino acid. Trends in Plant Science. 2010;15:89-97.

[18] Yang X, Liu YB, Qin LJ, et al. Trehalose-6-phosphate synthase gene TPS1 from Saccharomyces cerevisiae improve root growth in transgenic maize under drought stress. Plant Physiology Journal. 2015;51(3):363-369.

[19] Backhausen JE, Kitzmahn C,Scheibe R. Photosynth Res. 1994;42:75-86.

[20] Scheler C, Durner J, Astier J. Nitric oxide and reactive oxygenspecies in plant biotic interactions. Current Opinion in PlantBiology. 2013;16(4):534-539.

[21] Baxter A, Mittler R, Suzuki N. ROS as key players in plant stresssignalling. Journal of Experimental Botany. 2014;65(5):1229-1240.

[22] Sharma P, Jha AB, Dubey RS, et al. Reactive oxygen species, oxidative damage, and antioxidative defensemechanism in plants under stressful conditions. Journal of Botany. 2012; 2012:217037.

[23] Linton KJ. Structure and function of ABC transporters. Physiology. 2007;22(2):122-130.

[24] Yang Z, Dai Z, Lu R, et al. Transcriptome analysis of two species of jute in response to polyethylene glycol(PEG)- induced drought stress. Scientific Reports. 2017;7:16565.

[25] De Miguel M, Guevara MA, Sanchez-Gomez D, et al. Organ-specific metabolic responses to drought in Pinus pinaster Ait. Plant Physiology and Biochemistry. 2016;102:17-26.

[26] Hanf S, Fischer S, Hartmann H, et al. Online investigation of respiratory quotients in Pinus sylvestris and Picea abies during drought and shading by means of cavity-enhanced Raman multi-gas spectrometry. Analyst. 2015;140:4473-4481.

[27] Behringer D, Zimmermann H, Ziegenhagen B, et al. Differential gene expression reveals candidate genes for drought stress response in Abies alba (Pinaceae) [J]. PLo S One. 2015;10: e0124564.

[28] Matile P. Biochemistry of indian summer: physiology of autumnal leaf coloration. Exp Gerontol. 2000;35(2):145-158. 
[29] Seo PJ, Mas P. Stressing the role of the plant circadian clock. Trends Plant Sci. 2015;20(4): 230-237.

[30] Kiełbowicz-Matuk A, Rey P, Rorat T. Interplay between circadian rhythm, time of the day and osmotic stress constraints in the regulation of the expression of a Solanum double B-box gene. Annals of botany. 2014;113(5):831-842.

[31] Marcolino-Gomes J, Rodrigues FA, Fuganti-Pagliarini R, et al. Diurnal oscillations of soybean circadian clock and drought responsive genes. PLOS One. 2014;9 (1):e86402.

[32] Grabherr MG, Haas BJ, Yassour M, et al. Full-length transcriptome assembly from RNA-Seq data without a reference genome. Nature Biotechnology. 2011;29:644-652.

[33] Li B, Dewey C. RSEM: accurate transcript quantification from RNA-Seq data with or without a reference genome. BMC Bioinformatics. 2011;doi:10.1186/1471-2105-12-323.

[34] Young MD, Wakefield MJ, Smyth GK, et al. Gene ontology analysis for RNA-seq: accounting for selection bias. Genome Biology. 2010;doi:10.1186/gb-2010-11-2-r14.

[35] Kanehisa M, Araki M, Goto S, et al. KEGG for linking genomes to life and the environment. Nucleic Acids research. 2008;36:D480-D484.

[36] Mao X, Cai T, Olyarchuk JG, et al. Automated genome annotation and pathway identification using the KEGG Orthology (KO) as a controlled vocabulary. Bioinformatics. 2005;21:3787-3793.

[37] Livak KJ, Schmittgen TD. Analysis of relative gene expression data using real-time quantitative PCR and the $2^{-\square \square \mathrm{CT}}$ method. Methods. 2001;25:402-8.

\section{Figure Legends}

Table.1 Summary of transcriptome sequencing and assembly results for Medicago ruthenica

\begin{tabular}{cccccccc}
\hline Sample & Raw Reads & Clean reads & Clean bases & Error(\%) & Q20(\%) & Q30(\%) & GC(\%) \\
\hline A1 & 55346454 & 53622240 & $8.04 \mathrm{G}$ & 0.01 & 98.04 & 94.74 & 41.94 \\
\hline
\end{tabular}




\begin{tabular}{cccccccc}
\hline A2 & 69779438 & 66713486 & $10.01 \mathrm{G}$ & 0.01 & 97.71 & 93.85 & 41.70 \\
A3 & 53467816 & 51701418 & $7.76 \mathrm{G}$ & 0.01 & 98.02 & 94.70 & 41.82 \\
B1 & 54893996 & 52827488 & $7.92 \mathrm{G}$ & 0.01 & 97.84 & 94.40 & 41.68 \\
B2 & 50361132 & 48787544 & $7.32 \mathrm{G}$ & 0.01 & 98.05 & 94.78 & 41.57 \\
B3 & 47012036 & 44637736 & $6.7 \mathrm{G}$ & 0.01 & 97.75 & 94.28 & 41.79 \\
C1 & 57055692 & 54689354 & $8.2 \mathrm{G}$ & 0.01 & 97.87 & 94.45 & 41.79 \\
C2 & 46967170 & 45379566 & $6.81 \mathrm{G}$ & 0.01 & 97.95 & 94.70 & 41.91 \\
C3 & 46963384 & 45217556 & $6.78 \mathrm{G}$ & 0.01 & 97.70 & 94.20 & 41.74 \\
D1 & 58915238 & 56358828 & $8.45 \mathrm{G}$ & 0.01 & 97.88 & 94.50 & 41.61 \\
D2 & 56481880 & 54745144 & $8.21 \mathrm{G}$ & 0.01 & 98.00 & 94.64 & 41.87 \\
D3 & 56008960 & 54319404 & $8.15 \mathrm{G}$ & 0.01 & 98.07 & 94.80 & 41.87 \\
\hline
\end{tabular}

Note: The percentage of Q20 and Q30 are the proportion of nucleotides with a quality value $>20$ and > 30, respectively. The GC percentage presents the proportion of guanidine and cytosine nucleotides among total nucleotides. A1, A2 and A3 respectively represent three biological replicates of the leaf of non-stressed Medicago ruthenica; B1, B2 and B3 respectively represent three biological replicates of the leaf of Medicago ruthenica stressed 9d; C1, C2 and C3 respectively represent three biological replicates of the leaf of Medicago ruthenica stressed 12d; D1, D2, and D3 respectively represent three biological replicates of the leaf of Medicago ruthenica rehydration $4 \mathrm{~d}$.

Table.2 Primers for qRT-PCR

\begin{tabular}{ccc}
\hline Gene ID & & Primer (5' to 3') \\
\hline A(Cluster-60183.53665) & F:TCCCCTTGAACCATCCCTAACT & R:AACGGCTATTGACATGACACTGAT \\
B(Cluster20905.1) & F: TTGGCTCACGAATCTCACATACA & R: GCAAAGGACACAAAGGAGAGTAA \\
C(Cluster-60183.106139) & F: CAGATAGTCGTGAGGTGGTAATGTT & R: AGAGCCAAAGGTAGCGATGTG \\
D(Cluster-60183.827) & F: ACAGACGCAGCAGTGATAGT & R: GCAGAGGTGGAAGAGCATTATTG \\
E(Cluster-60183.11678) & F: AGTCCTTTACAGCATCAATGAACAG & R: GTTGAATCCACCACAGAGACCT
\end{tabular}



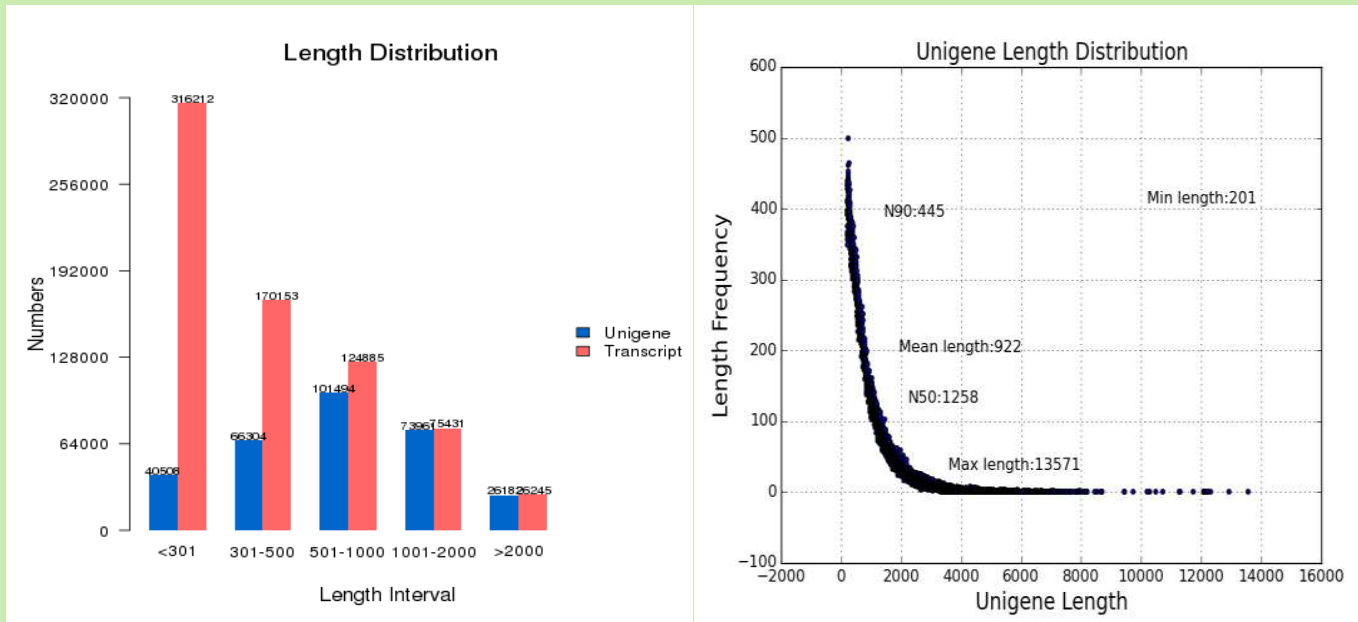

Figure.1 Length distribution of unigenes

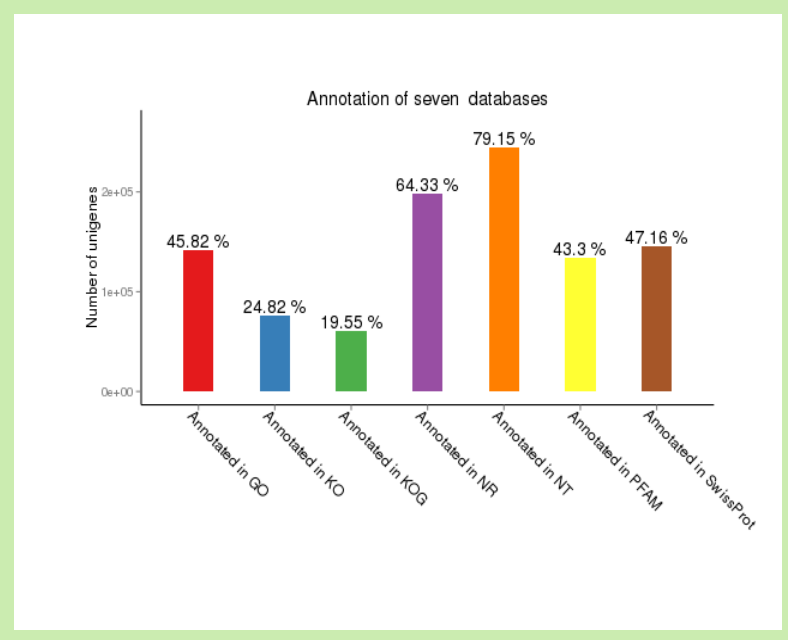

Figure.2 Statistics of gene annotation success rate 


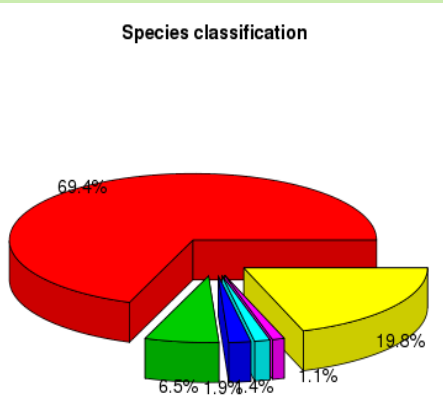

- Medicago truncatula - Cicer arietinum 믈 Gine soja
Hordeum vulgare
Similarity Distribution

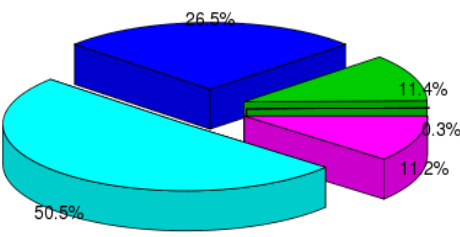

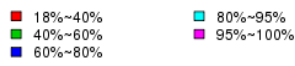

Figure.3 Species distribution of $\mathrm{Nr}$ annotation; similarity distribution of sequence
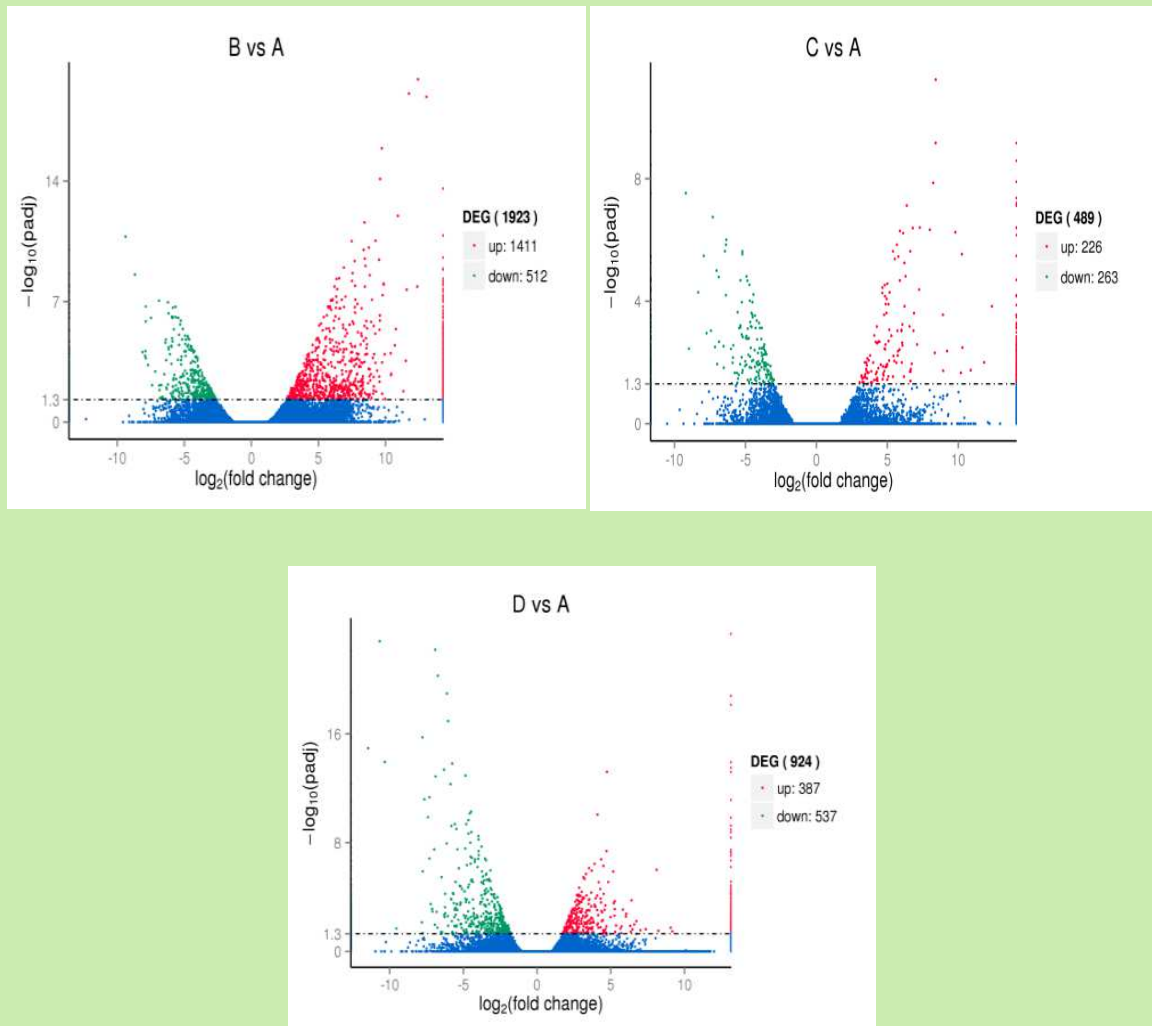

Figure.4 Volcano map of DEGs among different treatments

Note: A represents the control, B represents the drought stress 9d, C represents the drought stress 12d, D represents the rehydration, the same as below 


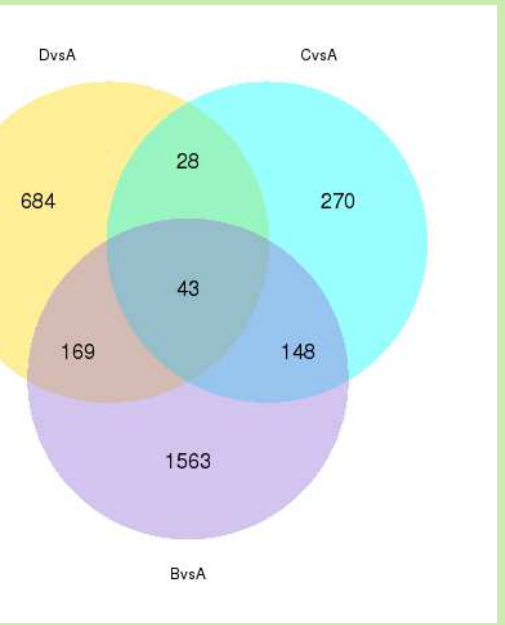

Figure.5 Venn diagram of DEGs in different treatment

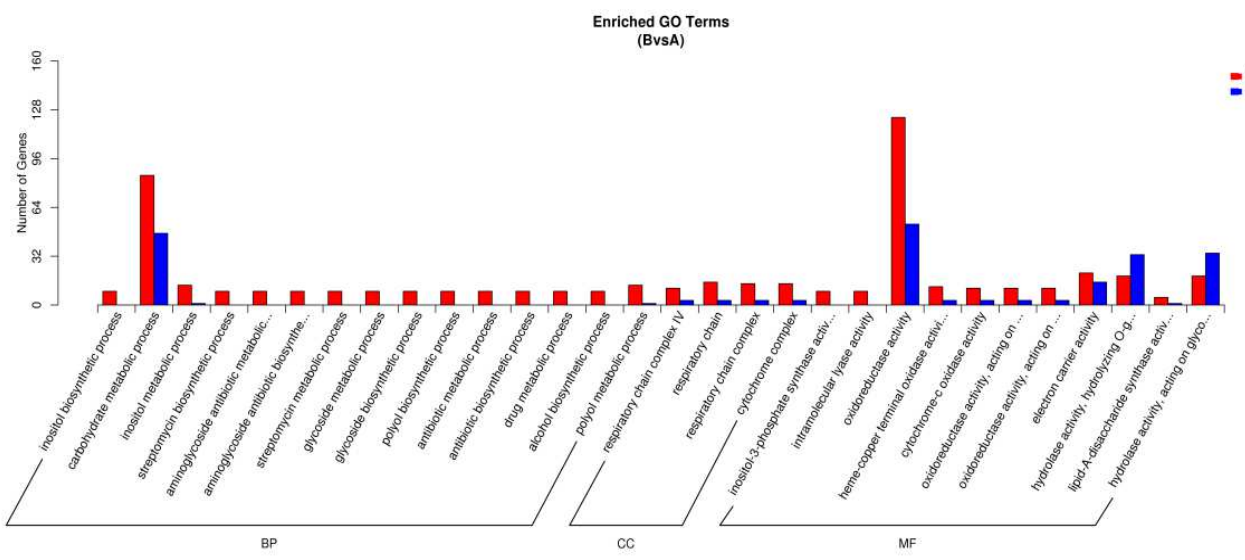




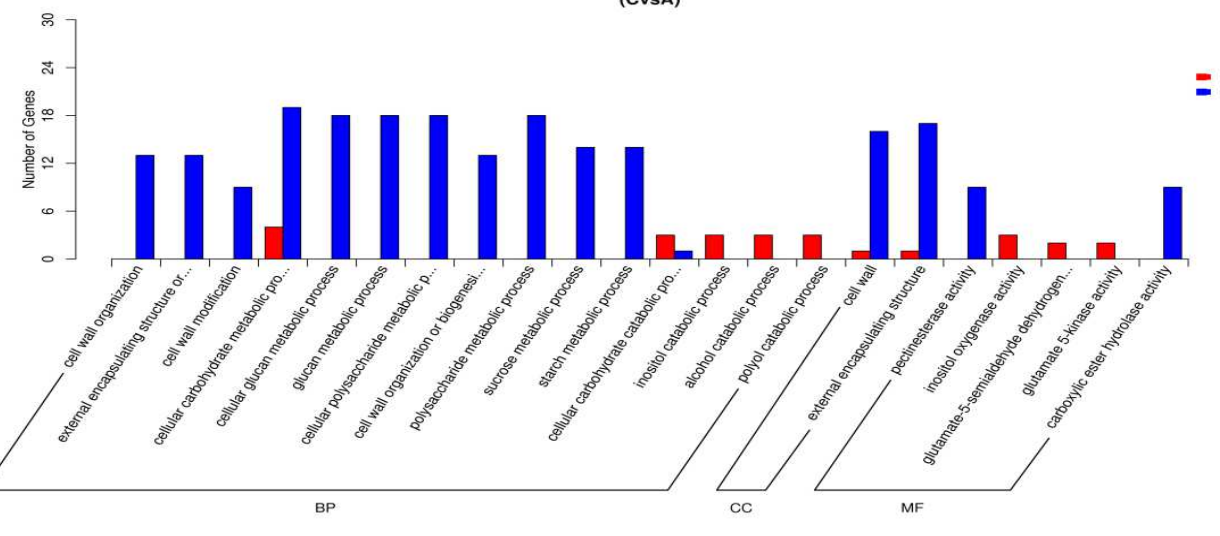

= up ${ }_{\text {Down }}$

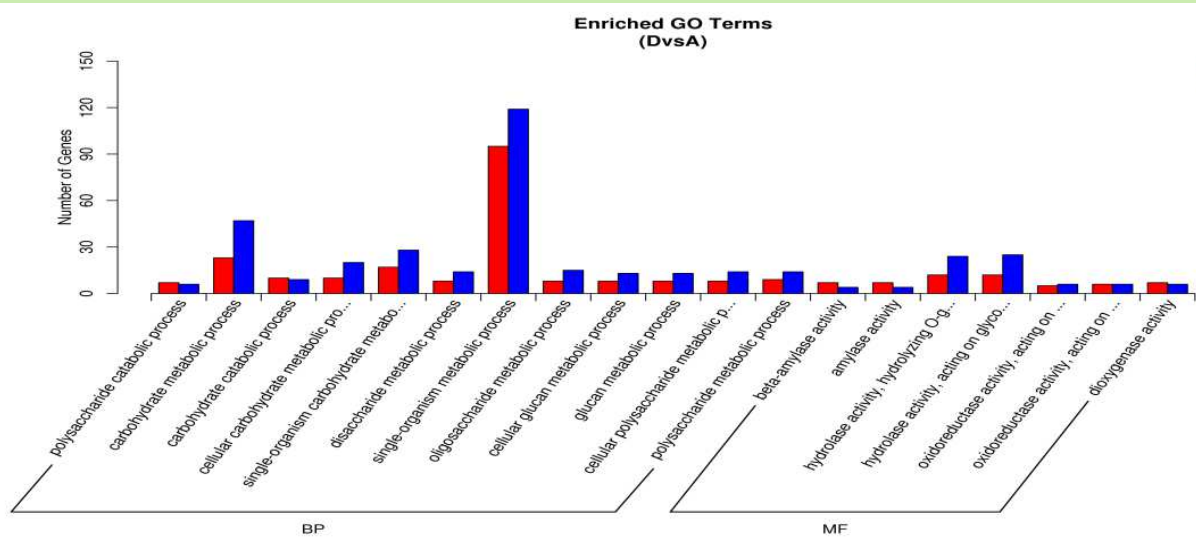

= 品own

Figure.6 Function annotation of differentially expressed genes in drought stress and rehydration 


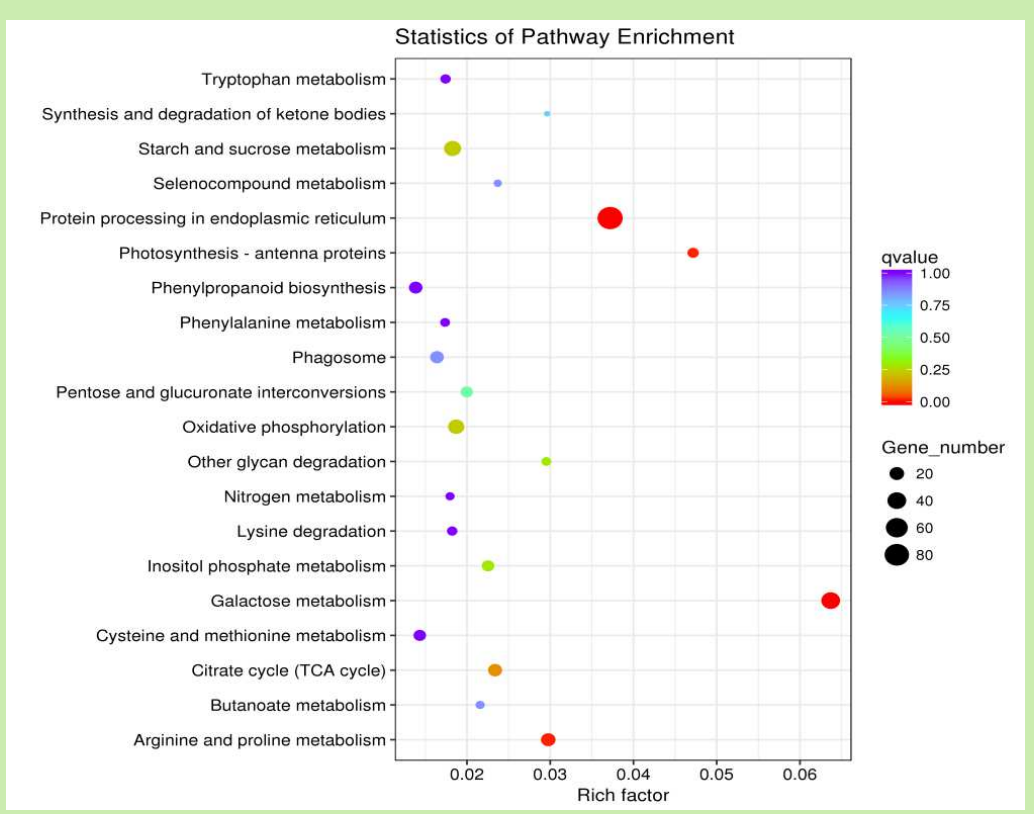

Figure.7 KEGG annotation of different genes under drought strss 9d

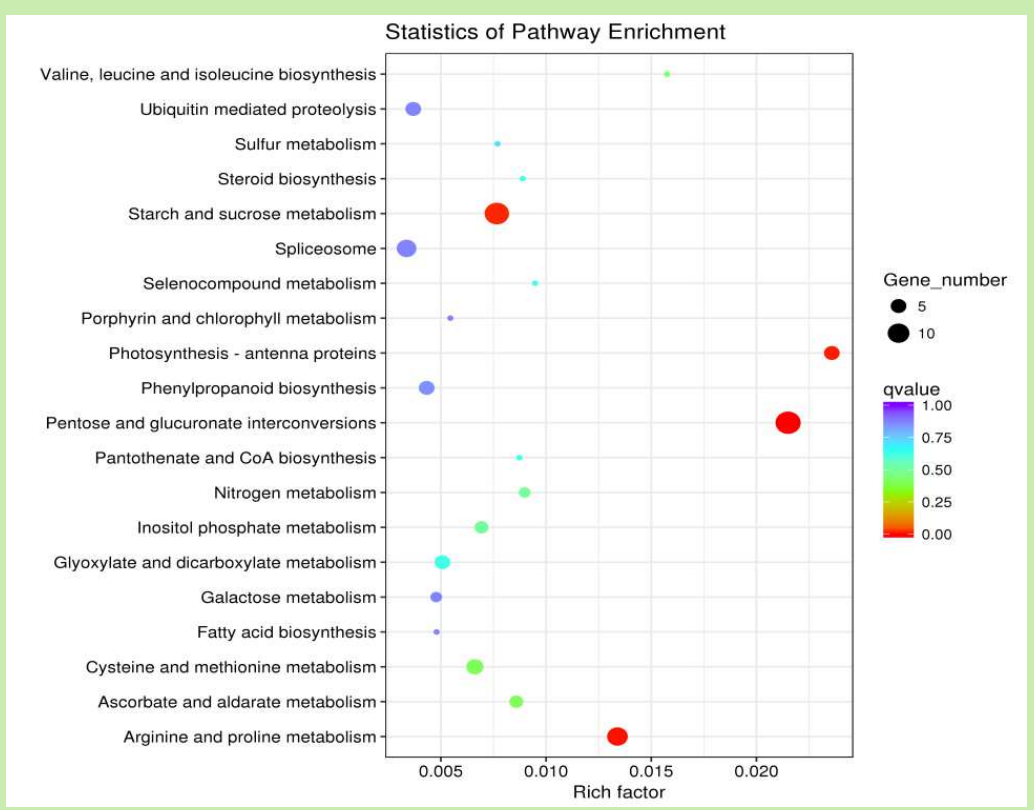

Figure.8 KEGG annotation of different genes under drought strss $12 \mathrm{~d}$ 


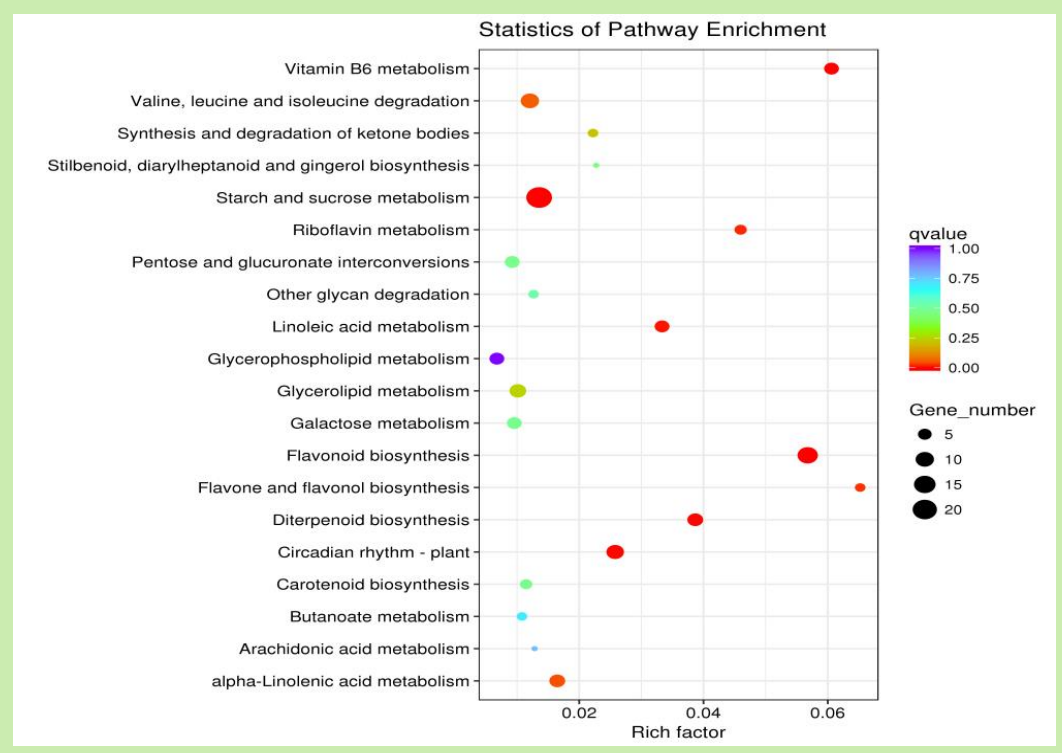

Figure.9 KEGG annotation of different genes under rehydration
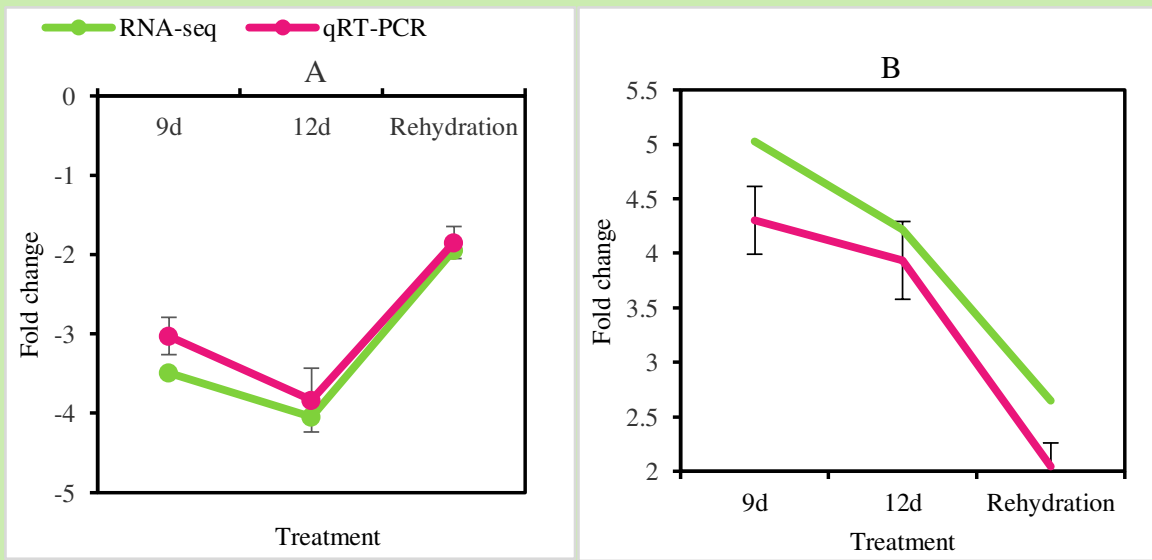

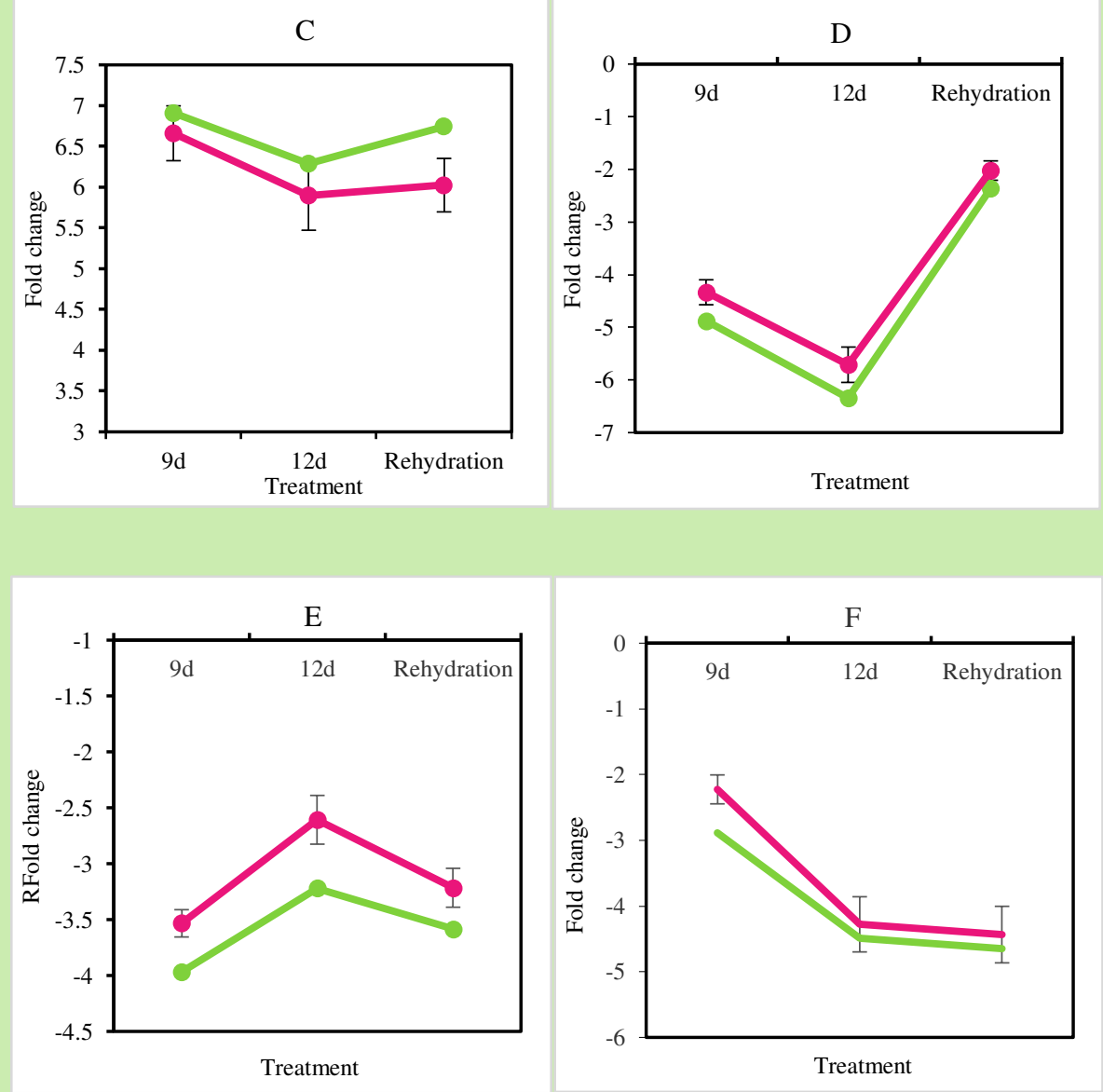

Figure 10. qRT-PCR result of differentially expressed genes 
Figures
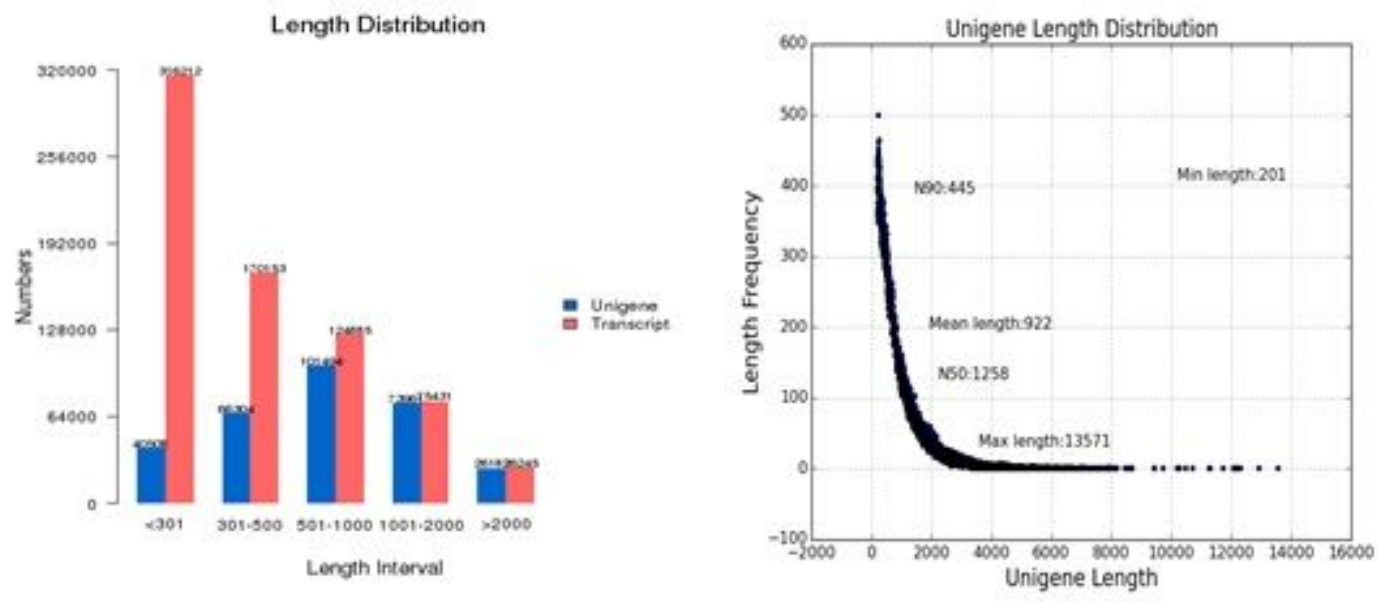

\section{Figure 1}

Length distribution of unigenes

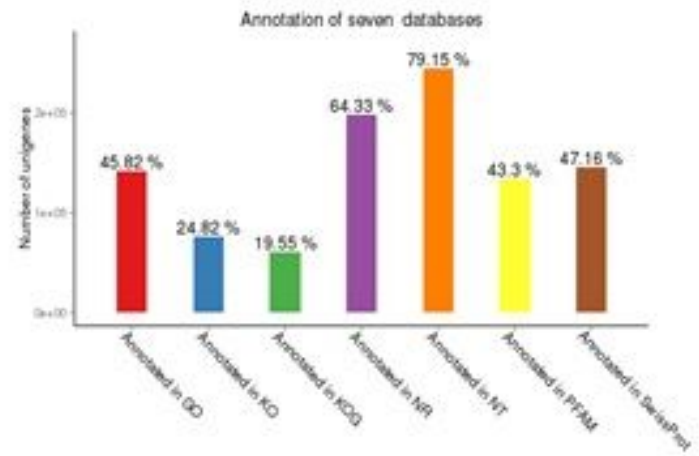

Figure 2

Statistics of gene annotation success rate 


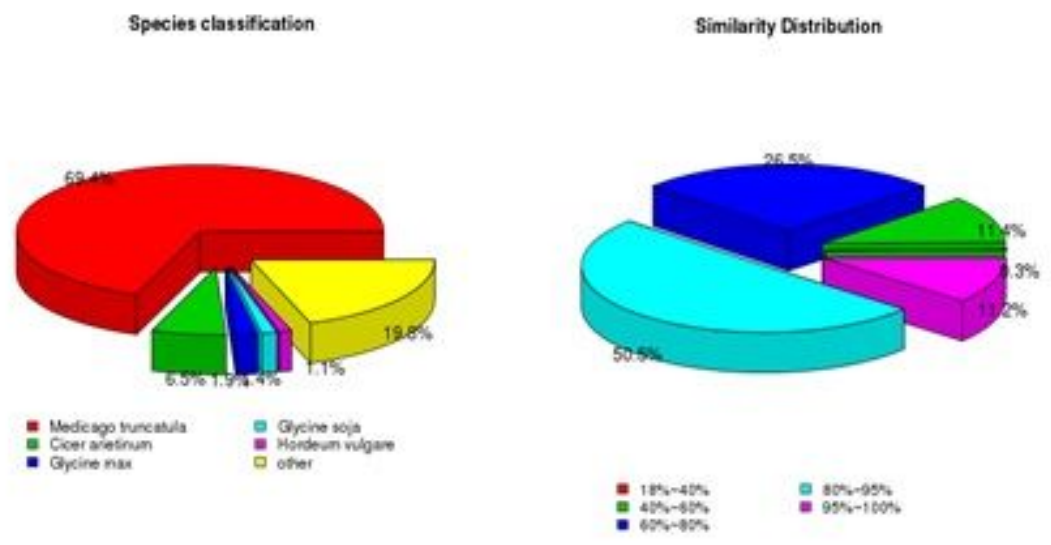

\section{Figure 3}

Species distribution of $\mathrm{Nr}$ annotation; similarity distribution of sequence
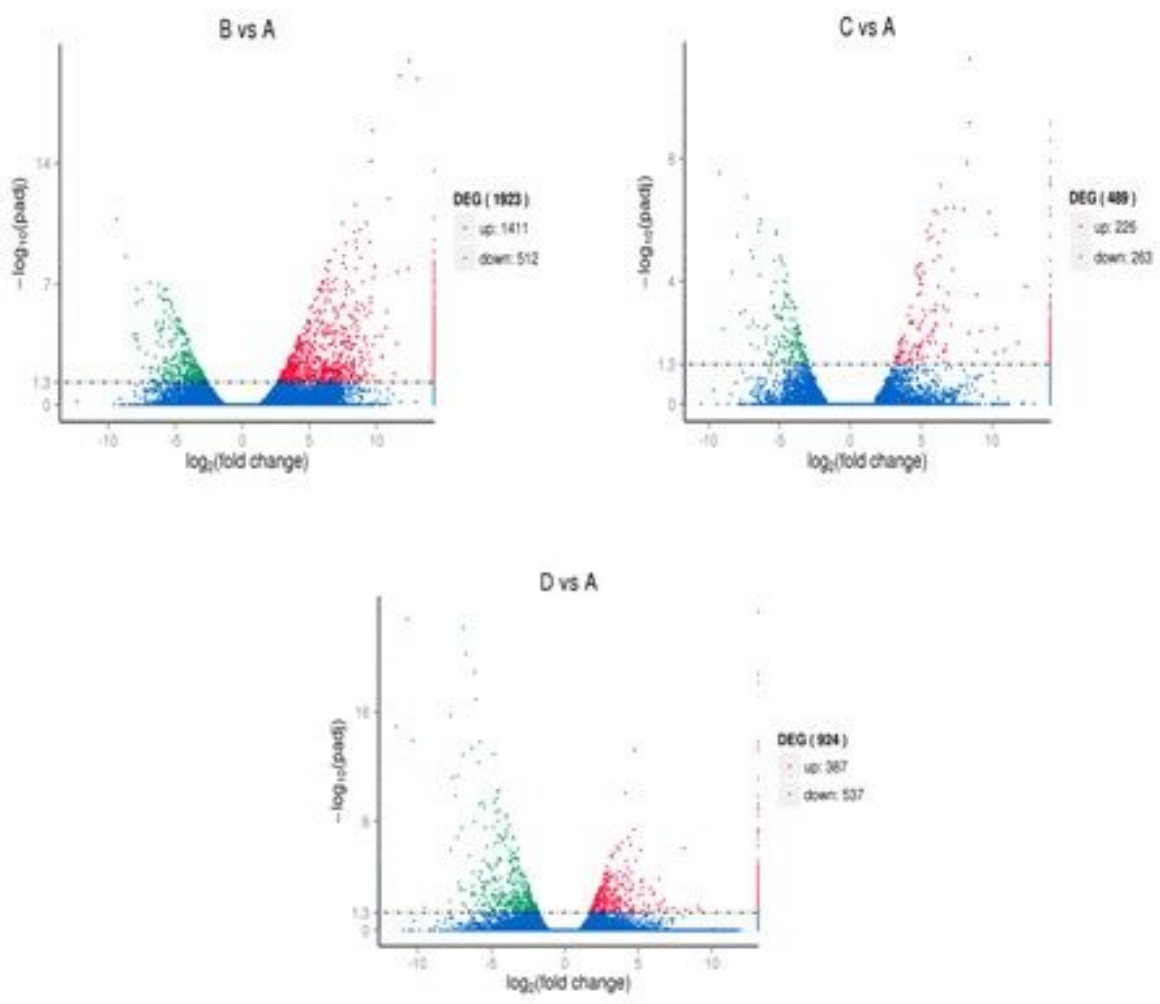

\section{Figure 4}

Volcano map of DEGs among different treatments Note: A represents the control, B represents the drought stress $9 d, C$ represents the drought stress $12 d$, D represents the rehydration, the same as below 


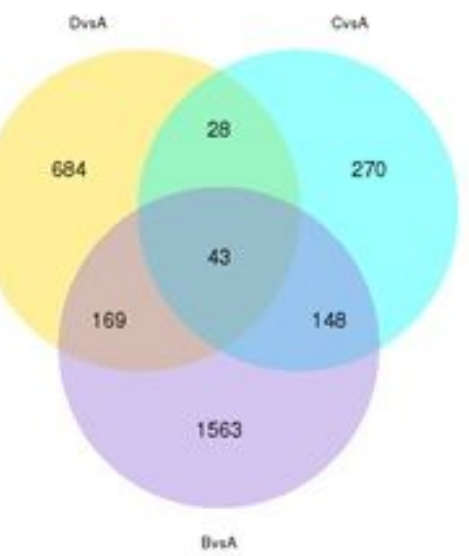

Figure 5

Venn diagram of DEGs in different treatment 

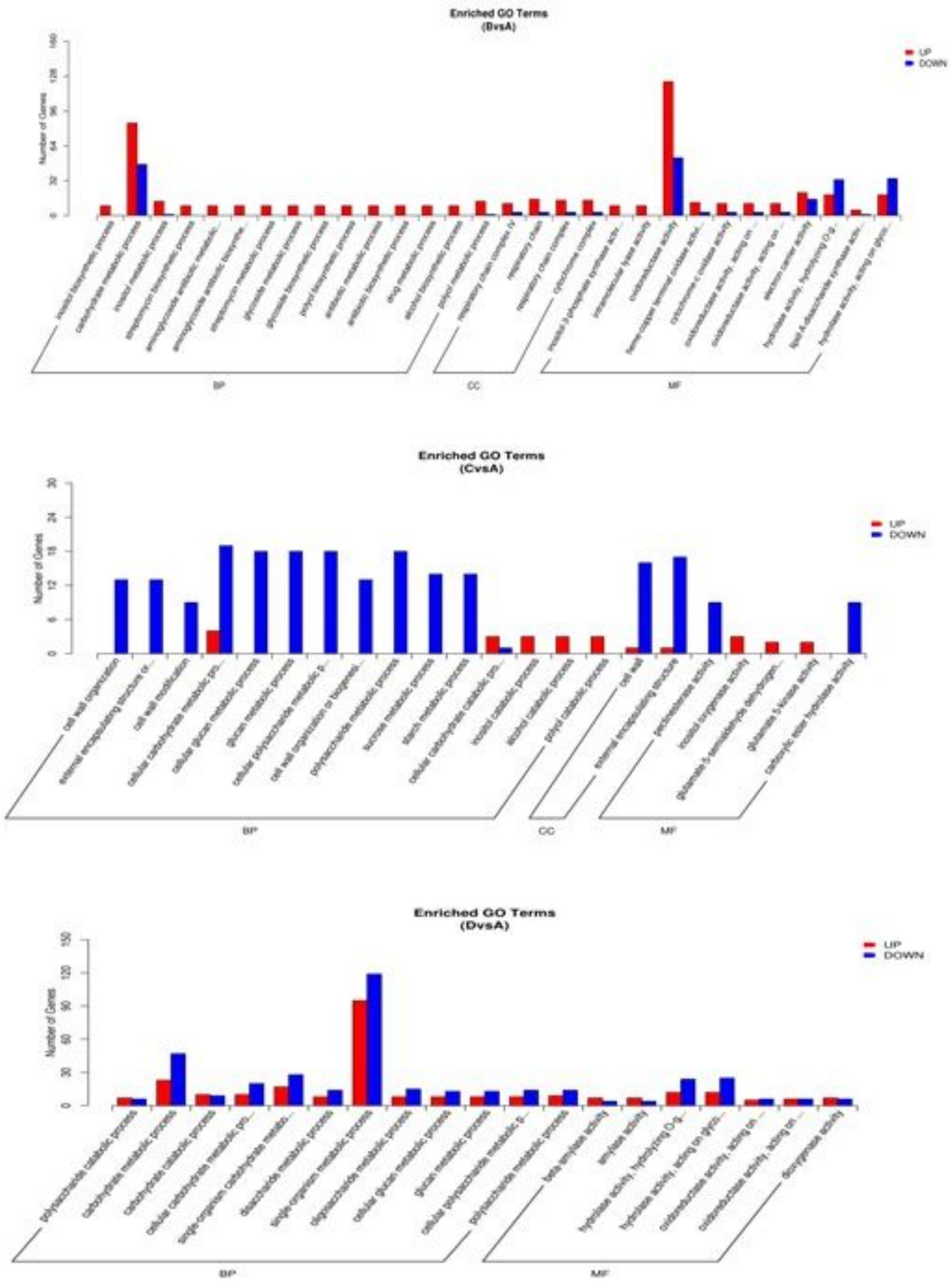

Figure 6

Function annotation of differentially expressed genes in drought stress and rehydration 


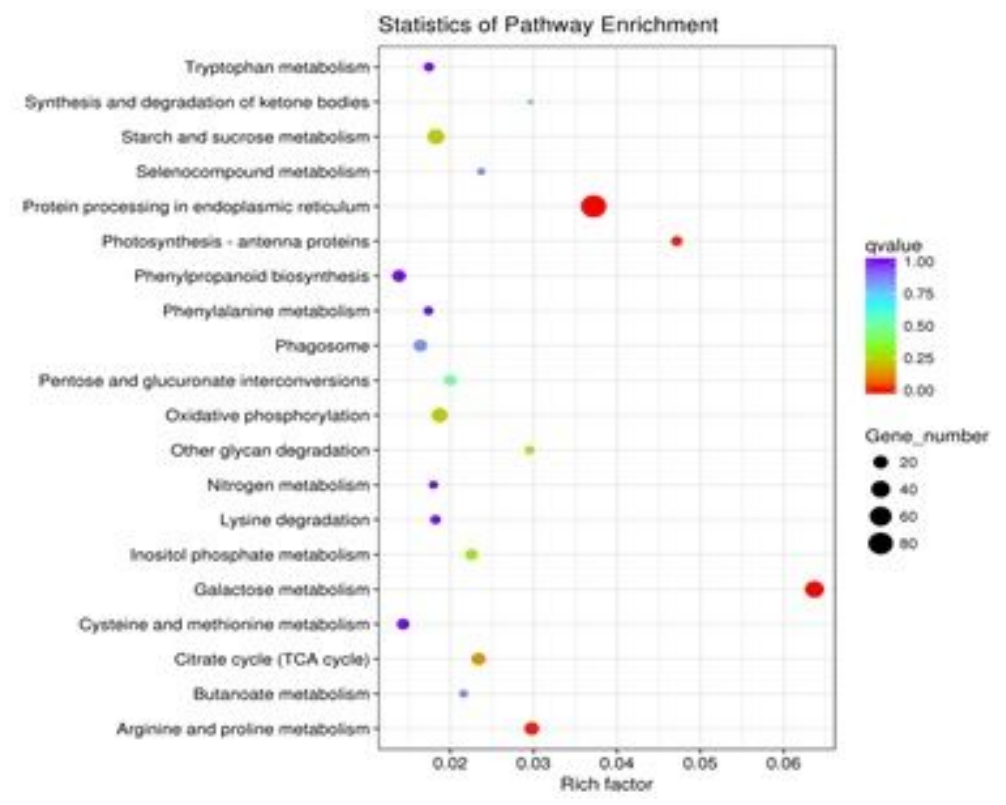

Figure 7

KEGG annotation of different genes under drought strss 9d

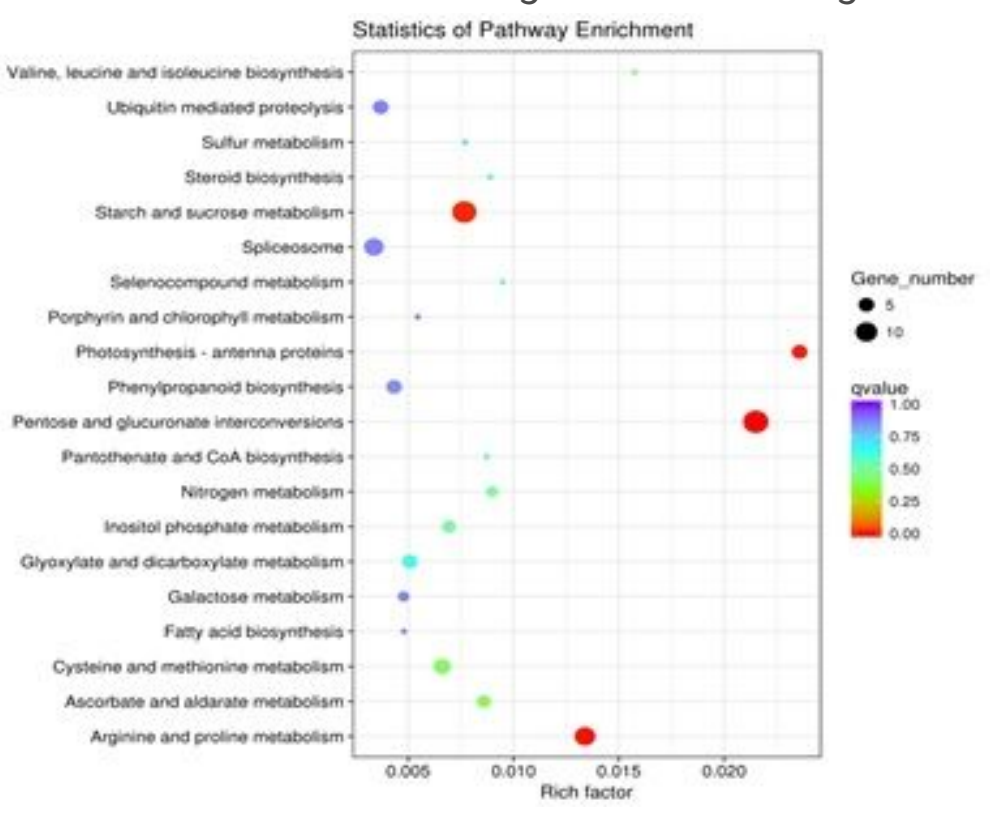

Figure 8

KEGG annotation of different genes under drought strss $12 d$ 


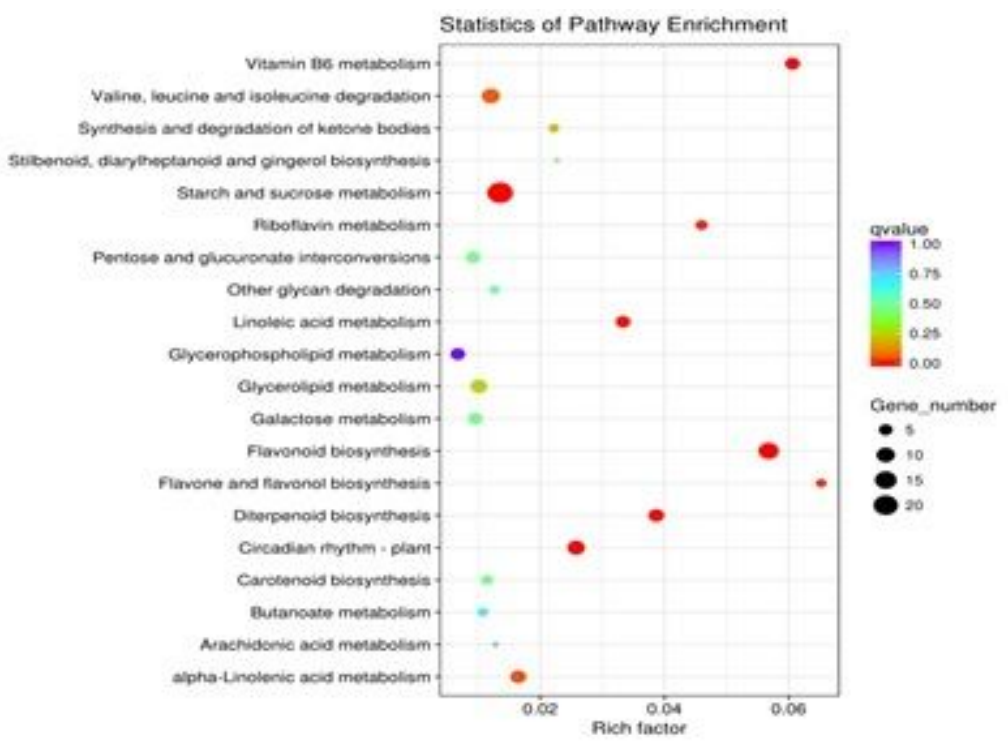

Figure 9

KEGG annotation of different genes under rehydration 

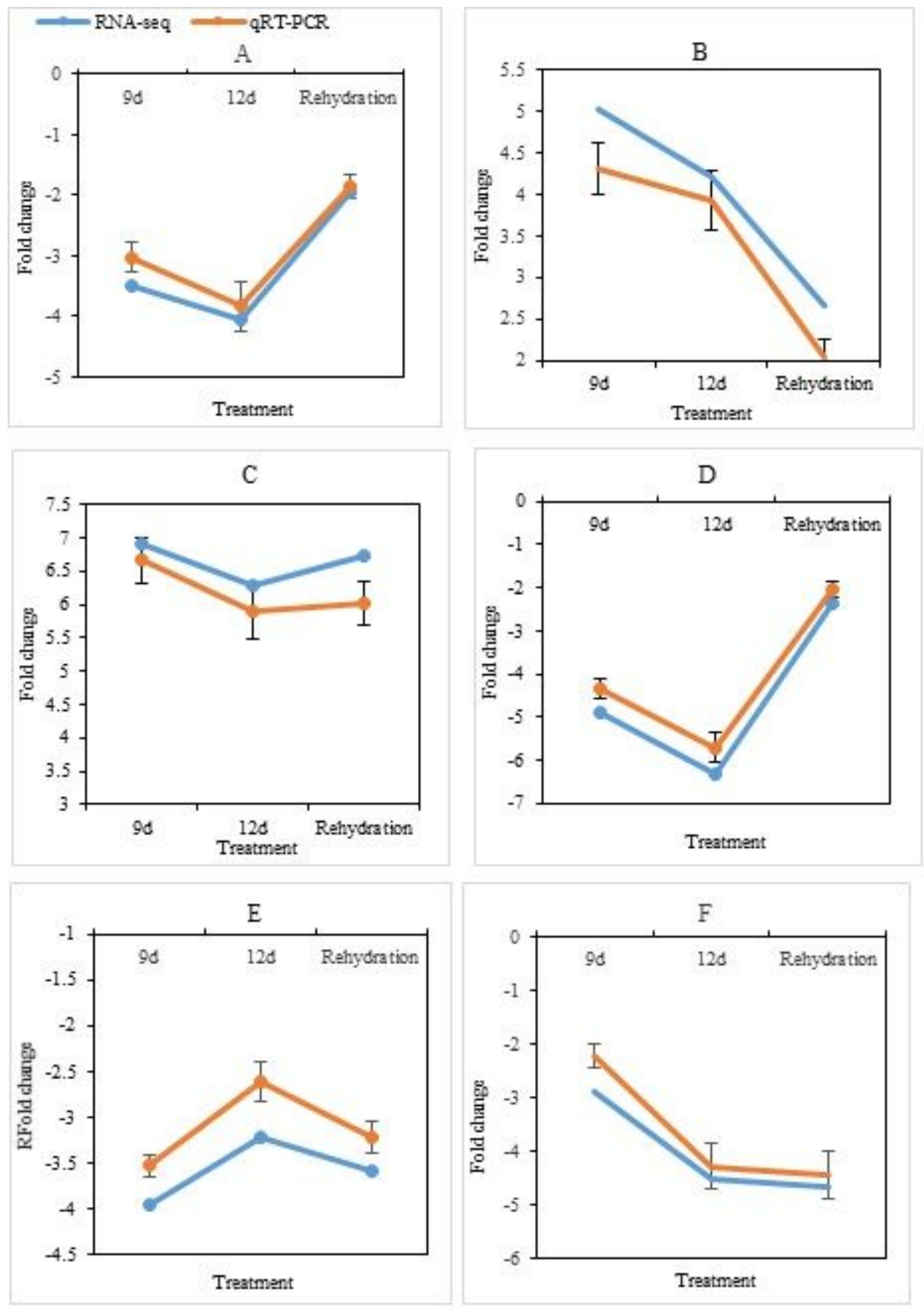

Figure 10

qRT-PCR result of differentially expressed genes

\section{Supplementary Files}

This is a list of supplementary files associated with this preprint. Click to download.

- Additionalfile.zip 